\title{
Introducing "Arithmetic Calculus" with Some Applications: New Terms, Definitions, Notations and Operators
}

\author{
Rahman Khatibi \\ Consultant Mathematical Modeller, Swindon, UK \\ Email: Rahman khatibi@yahoo.co.uk
}

Received 3 September 2014; revised 25 September 2014; accepted 10 October 2014

Copyright (C) 2014 by author and Scientific Research Publishing Inc.

This work is licensed under the Creative Commons Attribution International License (CC BY). http://creativecommons.org/licenses/by/4.0/

c. (i) Open Access

\begin{abstract}
New operators are presented to introduce "arithmetic calculus", where 1) the operators are just obvious mathematical facts, and 2) arithmetic calculus refers to summing and subtracting operations without solving equations. The sole aim of this paper is to make a case for arithmetic calculus, which is lurking in conventional mathematics and science but has no identity of its own. The underlying thinking is: 1) to shift the focus from the whole sequence to any of its single elements; and 2) to factorise each element to building blocks and rules. One outcome of this emerging calculus is to understand the interconnectivity in a family of sequences, without which they are seen as discrete entities with no interconnectivity. Arithmetic calculus is a step closer towards deriving a "Tree of Numbers" reminiscent of the Tree of Life. Another windfall outcome is to show that the deconvolution problem is explicitly well-posed but at the same time implicitly ill-conditioned; and this challenges a misconception that this problem is ill-posed. If the thinking in this paper is not new, this paper forges it through a mathematical spin by presenting new terms, definitions, notations and operators. The return for these out of the blue new aspects is far reaching.
\end{abstract}

\section{Keywords}

Natural Numbers, Sequences, Combinatorics, New Operators, Arithmetic Calculus, Factorisation, Complexity

\section{Introduction}

Calculus plays a pivotal role in science and this is so successful that searching for other possible calculi seems irrelevant. This paper has the ambition of carving out a chunk from classical mathematics to reform it as a new 
calculus to be referred to as "arithmetic calculus" in distinction from classical calculus, to be referred to as "algebraic calculus". Arithmetic calculus makes use of the convolution theorem and new operators, which are in the domain of traditional algebra but do not have an overarching identity. The constituents of the new calculus are all floating within current mathematical practices but the aim is to collect them together and to brand them by a hallmark. In this process, the thinking is to change focus from the whole sequence to any of its single elements and then express any single element as the product of building blocks and rules. This paper introduces the very basics of the new calculus and some of its potentials. New terms are italicised throughout the paper to inculcate their introduction. These are further collected in Glossary for the ease of reference.

Arithmetic calculus presented in this paper covers new terms, definitions, notations and operators. Admittedly, operations are self-obvious but their outcomes are far-reaching and include the following: 1) The paper challenges a complete misconception in mathematics that the deconvolution problem is ill-posed; whereas it is shown that this problem is explicitly well-posed but at the same time implicitly ill-conditioned. 2) A range of operations in algebra are simply clumsy and conceal the inner structure of number sequences; whereas arithmetic calculus unearths their structures. 3) It transpires that a family of mathematical problems expressed in the form of a set of numbers, series or sequences can be factorised into the product of two sequences: a) building blocks and b) rules, where this factorisation is tacit or even explicit in algebra but they fail to capture the clarity offered by arithmetic calculus. 4) The building blocks may be transformed into other set of interoperable sequences. The paper is not preoccupied by novelty but focussed on reforming existing knowledge in a different mould.

Two fundamental operators are presented, which are associated with a number of distinct variations. These new operators are by no means complex but even the obvious has to be stated. These operators are elaborated later in this paper but a glimpse of them is given here as a preview: 1) direct products of the sequence or sets of numbers $\left\{\begin{array}{llll}n_{1} & n_{2} & \cdots & n_{i}\end{array}\right\}$ by the sequence $\left\{\begin{array}{llll}m_{1} & m_{2} & \cdots & m_{i}\end{array}\right\}$ is annotated and defined as:

$$
\left\{\begin{array}{llll}
n_{1} & n_{2} & \cdots & n_{i}
\end{array}\right\} \circ\left\{\begin{array}{llll}
m_{1} & m_{2} & \cdots & m_{i}
\end{array}\right\}=\left\{\begin{array}{llll}
n_{1} m_{1} & n_{2} m_{2} & \cdots & n_{i} m_{i}
\end{array}\right\}
$$

where the proposed symbol of $\circ$ represents the direct product rules; 2) convolution products of the sequence $\left\{\begin{array}{lll}n_{1} & n_{2} & n_{3}\end{array}\right\}$ by the sequence $\left\{\begin{array}{ll}m_{1} & m_{2}\end{array}\right\}$ is defined as:

$$
\begin{array}{ll}
\left\{\begin{array}{lll}
n_{1} & n_{2} & n_{3}
\end{array}\right\} \hat{\oplus}\left\{\begin{array}{ll}
m_{1} & m_{2}
\end{array}\right\} \\
=\left\{\begin{array}{llll}
n_{1} m_{1} & n_{1} m_{2}+n_{2} m_{1} & n_{2} m_{2}+n_{3} m_{1} & n_{3} m_{2}
\end{array}\right\}
\end{array}
$$

where the proposed symbol of $\hat{\oplus}$ is one variant to notate the convolution product rules. Notably, the product in this example is composed of $4(=3+2-1)$ number of elements.

Example 1.1. Consider the mundane operation of:

$$
\begin{aligned}
& \left(3-2 x+2 x^{2}+x^{4}-5 x^{5}\right)\left(-1+3 x^{2}+3 x^{5}\right) \\
& =-3+2 x+7 x^{2}-6 x^{3}+5 x^{3}+14 x^{4}-3 x^{6}-9 x^{7}+0 x^{8}+3 x^{9}-15 x^{10}
\end{aligned}
$$

The operations concerning (1.3.a) by conventional multiplication approaches can also be handled by Ztransform (see appropriate textbooks), both of which are laborious. So mathematics can cope with such problems and there is nothing unique with them but this example and similar operations can be transformed as follows:

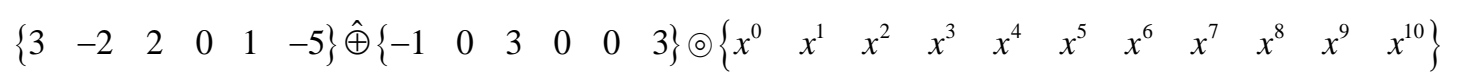

The approach inherent in (1.3.b) is systematic and automated in this paper, but at the expense of new definitions, new operators and new notations proposed by this paper yet to become standard. It is simply incredible that even simple operations are under the spell of tedious manual operations. This paper is a simple introduction and illustrates the new developments through examples but new theorems will be presented in the future.

The difference between algebraic calculus and arithmetic calculus is that the former leads normally to complex differential and/or integral equations describing a vast array of physical problems in space and time, e.g. Navier-Stokes equations describing turbulent mixing processes. In contrast, arithmetic calculus offers a more elementary calculus below the algebraic one and is to be focused on particulate media, which can describe evolutionary processes but not limited to these problems. 


\section{Literature Review}

Classical number series such as $\left\{\begin{array}{llllll}1^{m} & 2^{m} & 3^{m} & \cdots & n^{m}\end{array}\right\}$ are either treated by the theorem of binomial enumeration or by Stirling numbers, generating functions or other mathematical approaches. The theorem of binomial enumeration is often referred to as the calculus of finite differences or the umbral calculus, which studies the analogies between various sequences of polynomials $P_{m}$ and the powers sequence $n^{m}$. The subscript $m$ in $P_{m}$ was thought of as the shadow ("umbra" means "shadow" in Latin, whence the name umbral calculus) of the superscript $n$ in $n^{m}$. There are historical reviews on the subject, which are unnecessarily complex.

The calculus of number series comprising power series of natural numbers (e.g. numbers as

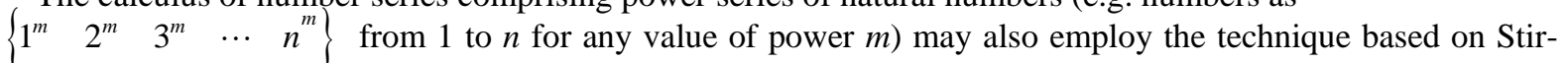
ling numbers of second kind. The background information is detailed in many textbooks. Stirling numbers of first and second kind or other sequences such as Bell numbers can turn out to be mathematically complex. Their details may be obtained from appropriate textbooks but there is no point in reviewing the literature on these subjects as they are top-down approaches and their underlying mathematics is classic but complex, varying notation and requiring intuition. The complexity is reflected by Wilf [1] on generating functions by remarking that a generating function: "after using it 4993 times it will seem quite routine". These top-down approaches are shrouded in mystery and ingenuity, which are useful as long as there is no alternative. This paper has the ambition of presenting an alternative but this is not the sole purpose of this paper. Missing arithmetic calculus is a gap, which adversely impacts science and engineering. Filling the gap is a driver for this paper.

\section{Terminology and Notations}

The strength of arithmetic calculus is in flexibility and in coping with a family of problems related to discrete and distinct objects. Basic definitions are given in this section.

\subsection{Refined Terms}

Definition 3.1. Sequences: A sequence of numbers is a collection of highly ordered set of individual numbers, each referred to as an element or a term, separated by a coma or space, e.g.:

$$
\{\mathbb{N}\}=\left\{\begin{array}{lllllll}
1 & 2 & 3 & \cdots & i & \cdots & n
\end{array}\right\}
$$

Series refer to sequences with specified operations between the elements, e.g.:

$$
\{\mathbb{N}\}={ }^{+}\left\{\begin{array}{llll}
1 & 2 & \cdots & n
\end{array}\right\}=\{1+2+3+\cdots+i+\cdots+n\}
$$

The pre-superscript “+” at the left hand side of curly brackets denotes the operations between the elements. A sequence is referred to a set when there is no obvious order in its elements and the same notation may be used.

Remark 3.1. Asequence may be basic, where all of its elements (or terms) are assumed independent and referred to as kernels. The proposed notations for kernels are:

$$
{ }^{\text {Kernel }}\{\mathbb{N}\}=\{\mathcal{K} \mid\{\mathbb{N}\}\}=\langle 1\rangle \text {, or more generally }\left\langle\begin{array}{lll}
a & b & \cdots\rangle
\end{array}\right.
$$

Kernels are elaborated later in the paper, showing that sequences are "primed" from a kernel. The notation $\{\cdot \mid \cdot\}$ is used extensively for a range of functionalities. The kernel of natural numbers is " 1 ".

Definition 3.2. Regeneration of Sequences: Regeneration is a distinct term proposed in this paper to carry out the very familiar operation of creating synthetic sequences by operating on the first $i$ elements recursively, where $i$ varies from 1 to a specific integer value. After the completion of the operations for all the terms, the regeneration gives rise to one new generation. There is no end to the ways of regeneration. This term should be contrasted with recursion defined below.

Definition 3.3. Recursion: This is also a form of regeneration but in its partial form, i.e. recursions do not restart from the first element as in regeneration. A classic example is Pascal Triangle or Fibonacci Numbers.

Definition 3.4. Generation ( $G$ and $g$ ): This creates a sense of the time dimension for the particulate phase of any entities but it is not cluck time as the beginning and end times of a generation are never specific. The operators for regeneration/recursion (defined in Section 4) maintain a sense of generation in the sequences.

Definition 3.5. Degree ( $D$ or $d$ ): The degree of a sequence stems from a specific way of multiplying two sequences together, as defined in Section 4. The degree of natural numbers is " 0 ". 
Definition 3.6. Order: by definition, Order $=d+g-1$.

Definition 3.7. Complexity: degree -1 refers to the complexity of a sequence or to the number of elements in a kernel. Therefore the complexity of natural numbers is " $1-1=0$ ".

Definition 3.8. Closed/Open Sequences: The number of the terms in closed sequences is finite but in open sequences, it is not.

Definition 3.9. Specification: The operators for regeneration and raising to powers are yet to be presented yet but they have a direct bearing on the order of a sequence. These three terms are not interchangeable, as each represents specific concepts in arithmetic calculus, which reflect the dimensionality in number sequences. The following notations are proposed:

$$
\underbrace{\left\{{\underset{w}{G=3}}_{\mid}^{\mathbb{Z}}\right\}^{4}}_{\varphi=4+3-1}
$$

(3.1.d) is specified as: Generation $G=3$, Degree $D=4$ and Order $\varphi=6$

$$
\{\text { Order / Degree } / \text { Generation } \mid \mathbb{Z}\}=\{\Phi / \mathcal{D} / \mathcal{G} \mid \mathbb{Z}\}=\varphi \text { or } G \text { or } D
$$

where "”" means or. The proposed terms of order and generation are general but degree should be replaced with the term exponent for a host of recursive sequences. The sequences of natural numbers are open-ended. Even if the focus is on size $n$ sequences, it can acquire any value and hence this sequence is open-ended. The above proposed notation is reminiscent of meteorologist notations showing multiple variables at the same location.

\subsection{Proposed Terms}

In arithmetic calculus, there are equivalents of integration and differentiation calculus and therefore it is proposed to call them conducement and reducement for special reasons.

Definition 3.10. Conducement: This is proposed in lieu of summing or integration. The infinitive conducement means: "to lead or tend, esp. with reference to a favourable or desirable result; to contribute; usually followed by to or toward" (http://dictionary.die.net/conduced). These connotations are appropriate, as the paper shows that summations lead to a more definitive outcome, each synthetic sequence may involve layers of conducement towards the required sequence and each conducement expresses the sum of its first $i$-terms.

Definition 3.11. Reducement: This refers to differentiation or differencing in arithmetic calculus. The verb "to reduce" is used widely in this paper but the infinitive reducement is not a dictionary word. This term is introduced here in lieu of differentiation; where the individual terms of a sequence at a lower order are obtained from higher orders, as a reverse/inverse operation of conducement.

Definition 3.12. Reducemental refers to a functional expression in arithmetic calculus, serving as the basic generator of a sequence.

\subsection{Notations for Conducement/Reducement}

The proposed notations for conducement and reducement are presented by the following two examples:

$$
\begin{aligned}
& \text { Cond }\{\mathbb{N}\}^{2}=\text { Cond }\{1 \quad 1\} \odot\left\{\left(\begin{array}{c}
n+1 \\
n-1
\end{array}\right)\left(\begin{array}{c}
n \\
n-2
\end{array}\right)\right\}=\left\{\begin{array}{lllllll}
1^{2} & 2^{2} & 3^{2} & \cdots & i^{2} & \cdots & n^{2}
\end{array}\right\} \\
& { }^{\text {Red }}\{\mathbb{N}\}^{2}=\left\{\mathcal{R} \mid\{\mathbb{N}\}^{2}\right\}=\left\{\begin{array}{ll}
1 & 1
\end{array}\right\} \odot\left\{\left(\begin{array}{c}
n+1 \\
n-1
\end{array}\right)\left(\begin{array}{c}
n \\
n-2
\end{array}\right)\right\}
\end{aligned}
$$

Remark 3.2. The two proposed terms of conducement and reducement and their derivatives together make a new philosophy, whereby 1) the focus from a sequence is shifted to any of its individual elements but the individual elements are readily transformable to the sequence, where this capability is in the fashion of "all for one and one for all;" 2) the individual elements are expressly described in terms of building blocks and rules.

Remark 3.3. The proposed term conducement is used in making new compound terms, such as conducemen- 
tal, conducemental algebra, conducemental sequences.

\section{Operators for Conducement-Direct Problems}

\subsection{Statement of the Problem}

There are two key problems in conventional algebra: direct prediction problems and inverse problems. Direct prediction problems often lead to the formulation of a system of equations, many of which have been shown to be well-posed (or properly-posed) problems, satisfying the three conditions of: 1) the solution exists; 2) the solution is unique (the number of equations is equal to the number of unknowns); and 3) the solution is stable (small changes in the measurement do not lead to large changes in the solution) by depending on the data. The conducement problem is the equivalent of the direct prediction problem and its operators are presented in this section, where there is no equation to be solved. Conducement problems are implemented as products of rules and building blocks, during which the products encapsulate (without retaining any clue on) the nature of the operation expressing them and therefore their reconstruction becomes intractable.

Multiplication rules, presented below, operate on the corresponding elements of two sequences. The products comprise a series of elements within which the preceding terms are nested as sub-terms; and summing the respective sub-terms produce the value of each term. The outcomes are equivalent to integration but there are differences warranting a different terminology. Consider the following two sequences:

$$
\begin{aligned}
& \left\{\begin{array}{lllll}
t_{1} & t_{2} & \cdots & t_{n-1} & t_{n}
\end{array}\right\} \\
& \left\{\begin{array}{lllll}
r_{1} & r_{2} & \cdots & r_{m-1} & r_{m}
\end{array}\right\}
\end{aligned}
$$

Both (4.1.a) and (4.1.b) can be sets of numbers or sequences. One of them acts as rules and the other as building blocks or invariants. The following multiplication rules are presented.

\subsection{Direct Products}

The direct product (or dot products) rule is defined as:

$$
\begin{aligned}
\left\{\begin{array}{lllll}
t_{1} & t_{2} & \cdots & t_{n-1} & t_{n}
\end{array}\right\} \circ\left\{\begin{array}{llllll}
r_{1} & r_{2} & \cdots & r_{n-1} & r_{n}
\end{array}\right\}=\left\{\begin{array}{llllll}
t_{1} r_{1} & t_{2} r_{2} & t_{3} r_{3} & \cdots & t_{n-1} r_{n-1} & t_{n} r_{n}
\end{array}\right\} \\
\left\{\begin{array}{lllllll}
t_{1} & t_{2} & \cdots & t_{n-1} & t_{n}
\end{array}\right\} \odot\left\{\begin{array}{llllll}
r_{1} & r_{2} & \cdots & r_{n-1} & r_{n}
\end{array}\right\}=\left\{\begin{array}{llll}
t_{1} r_{1}+t_{2} r_{2}+t_{3} r_{3}+\cdots+t_{n-1} r_{n-1}+t_{n} r_{n}
\end{array}\right\}
\end{aligned}
$$

In (4.2.a), dot products are denoted by " $\circ$ " but the regeneration from the first element in (4.2.b) is denoted by “ $\odot$ ”. Note that the reducement in (3.2.a) and (3.2.b) employs the regeneration operation denoted by “ $\odot$ ” and this remove the need for the usual summation notation within the ranging of variation of $n$.

Example 4.1. The examples below illustrate a glimpse of the utility of (4.2.a)-(4.2.b)

$$
\begin{aligned}
& \left\{\begin{array}{lllll}
1 & 2 & 3 & \cdots & n
\end{array}\right\} \circ\left\{\begin{array}{lllll}
1 & 2 & 3 & \cdots & n
\end{array}\right\}=\left\{\begin{array}{lllll}
1^{2} & 2^{2} & 3^{2} & \cdots & n^{2}
\end{array}\right\}
\end{aligned}
$$

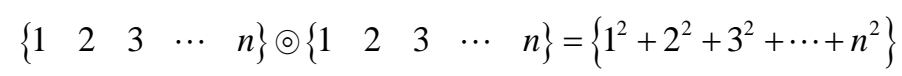

\subsection{Regeneration Products}

The regenerating product rule is defined as:

$$
\begin{aligned}
& \left\{\begin{array}{lllll}
t_{1} & t_{2} & \ldots & t_{n-1} & t_{n}
\end{array}\right\} \oplus\left\{\begin{array}{lllll}
r_{1} & r_{2} & \ldots & r_{m-1} & r_{m}
\end{array}\right\}=\left\{\begin{array}{ccc} 
& & t_{1} \\
t_{1} & t_{1} & \\
& t_{2} & \ldots \\
t_{2} & t_{3}
\end{array}\right\} \oplus\left\{\begin{array}{llll} 
& & r_{1} & \\
& r_{1} & r_{2} & \ldots \\
r_{1} & r_{3}
\end{array}\right\}
\end{aligned}
$$

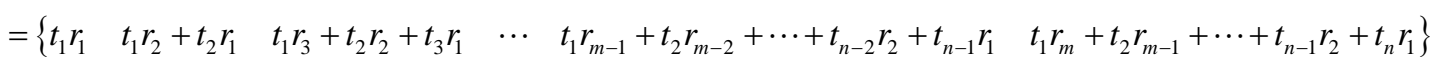

The number of terms in the product is equal to the largest value of $m$ or $n$. There are many problems, in which regenerating products can be identified. The constituent sequences for the product make up three cases as illustrated below by examples:

Case 1: Both sequences are closed, e.g.: 


$$
\begin{aligned}
& \left\{\begin{array}{llll}
1 & 3 & 3 & 1
\end{array}\right\} \oplus\left\{\begin{array}{lll}
1 & 2 & 1
\end{array}\right\}=\left\{\begin{array}{llll}
1 \times 1 & 1 \times 2+3 \times 1 & 1 \times 1+3 \times 2+3 \times 1 & 3 \times 1+3 \times 2+1 \times 1
\end{array}\right\} \\
& =\left\{\begin{array}{llll}
1 & 5 & 10 & 10
\end{array}\right\}
\end{aligned}
$$

Case 2: One sequence is open and another one closed, e.g.:

$$
\begin{aligned}
\left\{\begin{array}{llllll}
1 & 2 & 3 & 4 & 5 & \cdots
\end{array}\right\} \oplus\left\{\begin{array}{rl}
1 & 1
\end{array}\right\} & =\left\{\begin{array}{llllll}
1 \times 1 & 1 \times 1+2 \times 1 & 2 \times 1+3 \times 1 & \cdots
\end{array}\right\} \\
& =\left\{\begin{array}{llllll}
1 & 3 & 5 & 7 & 9 & \cdots
\end{array}\right\}
\end{aligned}
$$

which is the arithmetic progression sequence of natural numbers.

Case 3: Both sequences are open. Consider two unitary sequences:

$$
\begin{aligned}
& \left\{\begin{array}{llllll}
1 & 1 & 1 & 1 & \cdots
\end{array}\right\} \oplus\left\{\begin{array}{lllll}
1 & 1 & 1 & 1 & \cdots
\end{array}\right\} \\
& =\left\{\begin{array}{llllll}
\overbrace{1 \times 1}^{\text {Term } 1} & \overbrace{1 \times 1+1 \times 1}^{\text {Term } 2} & \cdots & \overbrace{1 \times 1+1 \times 1+1 \times 1+1 \times 1} & \cdots
\end{array}\right\} \\
& =\left\{\begin{array}{llllll}
1 & 2 & 3 & 4 & \cdots
\end{array}\right\}
\end{aligned}
$$

The term regeneration is not a standard one but this operator is hidden in many standard sequences.

\subsection{Convolution Products}

The convoluting product rule is defined as:

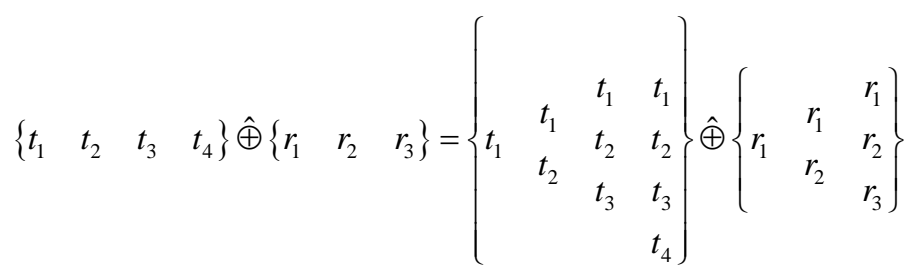

$$
\begin{aligned}
& =\left\{\begin{array}{llllll}
t_{1} r_{1} & t_{1} r_{2}+t_{2} r_{1} & t_{1} r_{3}+t_{2} r_{2}+t_{3} r_{1} & t_{2} r_{3}+t_{3} r_{2}+t_{4} r_{1} & t_{3} r_{2}+t_{4} r_{1} & t_{4} r_{1}
\end{array}\right\}
\end{aligned}
$$

The last two terms: $t_{3} r_{2}+t_{4} r_{1}$ and $t_{4} r_{1}$, cause dipping in the trailing values of the product, as may be seen in the example below. Three cases are possible. Notably, (1.3.a)-(1.3.b) can now be easily worked out.

Case 1: Both sequences are closed, as in the following example:

$$
\begin{aligned}
& \left\{\begin{array}{llll}
1 & 3 & 3 & 1
\end{array}\right\} \hat{\oplus}\left\{\begin{array}{lll}
1 & 2 & 1
\end{array}\right\}
\end{aligned}
$$

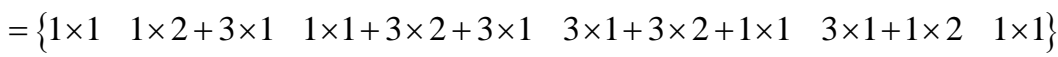

$$
\begin{aligned}
& =\left\{\begin{array}{llllll}
1 & 5 & 10 & 10 & \underbrace{5}_{\text {dipping }} 1
\end{array}\right\}
\end{aligned}
$$

Hence, the notation, $\hat{\oplus}$, reflects the dipping at the trailing elements.

Case 2: One sequence is open and the other one is closed, as in the case of regenerating products.

Case 3: Both sequences are open, but in reality this is meaningless.

Remark 4.1. The difference between the respective multiplications is in the trailing dipping terms. In regenerating products, the trailing terms are curtailed but they dip in the convoluting products and this message is conveyed by the symbol of over-hat.

Remark 4.2. This operator has similarities with the convolution theory and hence the term, originally given by Volttera in 1913, described as follows:

$$
(f \cdot g)(t)=\int_{-\infty}^{\infty} f(\tau) g(t-\tau) \mathrm{d} \tau
$$

\subsection{A Step towards Deconvolution}

Division of two sequences is one step towards deconvolution or the inverse problems of conducemental algebra, where the term deconvolution is used in its normal sense of mathematic. The operation is as follows: 


$$
\left\{\begin{array}{llll}
t_{1} & t_{2} & t_{3} & t_{4}
\end{array}\right\} \oslash\left\{\begin{array}{lll}
r_{1} & r_{2} & r_{3}
\end{array}\right\}=\frac{\left\{\begin{array}{llll}
t_{1} & t_{2} & t_{3} & t_{4}
\end{array}\right\} \oplus\left\{\begin{array}{llll}
1 & 0 & 0 & 0
\end{array}\right\}}{\left\{\begin{array}{lllll}
r_{1} & r_{2} & r_{3}
\end{array}\right\} \oplus\left\{\begin{array}{lll}
1 & 0 & 0
\end{array}\right\}}=\frac{\left\{\begin{array}{llll}
t_{1} & t_{2} & t_{3} & t_{4}
\end{array}\right\}}{\left\{\begin{array}{llll}
1 & 0 & 0 & 0
\end{array}\right\}} \frac{\left\{\begin{array}{lll}
1 & 0 & 0
\end{array}\right.}{\left\{\begin{array}{llll}
r_{1} & r_{2} & r_{3}
\end{array}\right\}}
$$

where “ $\oslash$ " is proposed to denote division. The above is evident as:

By definition:

$$
\begin{aligned}
& \{1\}=\left\{\begin{array}{ll}
1 & 0
\end{array}\right\}=\left\{\begin{array}{lll}
1 & 0 & 0
\end{array}\right\}=\left\{\begin{array}{llll}
1 & 0 & 0 & 0
\end{array}\right\}=\left\{\begin{array}{lllll}
1 & 0 & 0 & 0 & \cdots
\end{array}\right\} \\
& \left\{\begin{array}{lll}
r_{1} & r_{2} & r_{3}
\end{array}\right\} \oplus\left\{\begin{array}{lll}
1 & 0 & 0
\end{array}\right\}=\left\{\begin{array}{lll}
r_{1} \times 1 & r_{1} \times 0+r_{2} \times 1 & r_{1} \times 0+r_{2} \times 0+r_{3} \times 1
\end{array}\right\}=\left\{\begin{array}{lll}
r_{1} & r_{2} & r_{3}
\end{array}\right\} \\
& \left\{\begin{array}{llll}
t_{1} & t_{2} & t_{3} & t_{4}
\end{array}\right\} \oplus\left\{\begin{array}{llll}
1 & 0 & 0 & 0
\end{array}\right\}=\left\{\begin{array}{llll}
t_{1} & t_{2} & t_{3} & t_{4}
\end{array}\right\}
\end{aligned}
$$

There is nothing unique about: $\frac{\left\{\begin{array}{lll}1 & 0 & 0\end{array}\right\}}{\left\{\begin{array}{lll}r_{1} & r_{2} & r_{3}\end{array}\right\}}$, but its treatment through conducemental algebra is presented in Section 5 in more detail as the inverse problem.

\subsection{Algebraic Products}

The algebraic product rule is defined as:

$$
\begin{aligned}
& \left\{\begin{array}{lllll}
t_{1} & t_{2} & t_{3} & \cdots & t_{n}
\end{array}\right\} \otimes\left\{\begin{array}{lllll}
r_{1} & r_{2} & r_{3} & \cdots & r_{n}
\end{array}\right\}= \\
& \left\{t_{1} r_{1}+t_{1} r_{2}+t_{1} r_{3}+\cdots \cdots+t_{1} r_{n} \quad t_{2} r_{1}+t_{2} r_{2}+t_{2} r_{3}+\cdots+t_{2} r_{n} \quad t_{3} r_{1}+t_{3} r_{2}+t_{3} r_{3}+\cdots+t_{3} r_{n} \quad \cdots \quad t_{n} r_{1}+t_{n} r_{2}+t_{n} r_{3}+\cdots+t_{n} r_{n}\right\}
\end{aligned}
$$

The above is illustrated as:

$$
\left\{\begin{array}{lll}
1 & 2 & 3
\end{array}\right\} \otimes\left\{\begin{array}{lll}
1 & 2 & 3
\end{array}\right\}=\{1 \times 1+1 \times 2+1 \times 3 \quad 2 \times 1+2 \times 2+2 \times 3 \quad 3 \times 1+3 \times 2+3 \times 3\}={ }^{+}\left\{\begin{array}{lll}
1 & 2 & 3
\end{array}\right\}^{2}
$$

\subsection{Deeper Understanding of Convolution Products}

Mathematical operations are normally carried out in the algebraic space without any designated notation for convolution products, although the convolution theory is well established. The aim is to unearth the operations by conducemental algebra. Consider the sequence of natural numbers (Order 1 and Generation 2). It is regenerated to its Generation 3, as a new sequence, by regenerating the first $i$-number of terms as:

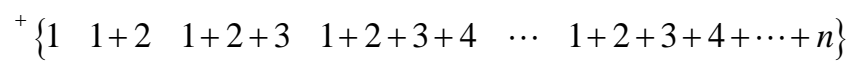

where the sum of each of the regenerated term based on conventional algebra of natural numbers is:

$$
n(n+1) / 2
$$

The alternative expression for the $n^{\text {th }}$ element is written as:

$$
\ddot{1} \times \bar{n}+\ddot{2} \times \overline{(n-1)}+\ddot{3} \times \overline{(n-2)}+\cdots+(\ddot{n-1)} \times \overline{2}+\ddot{n} \times \overline{1}
$$

The hidden format of (4.11.c) is interesting, as over-bars hint that the expression is a product of two sequences of natural numbers, one in the increasing order but the other is its reverse. Conventional algebra is poor for shielding this but arithmetic calculus exploits it, as follows:

$$
\text { Factorise (4.11.c) }=\left\{\begin{array}{lllllll}
1 & 2 & \cdots & i & \cdots & n-1 & n
\end{array}\right\} \odot\left\{\begin{array}{lllllll}
n & n-1 & \cdots & i & \cdots & 2 & 1
\end{array}\right\}
$$

Now, (4.11.d) reveals the structure of the product by factorisation but expressing sequences in the reverse order is counterintuitive. Hence the paper successfully uses an alternative approach to rectify the sequence in reverse order:

$$
\begin{aligned}
& \left\{\begin{array}{lllllll}
1 & 2 & \cdots & i & \cdots & n-1 & n
\end{array}\right\} \odot\left\{\begin{array}{lllllll}
n & n-1 & \cdots & i & \cdots & 2 & 1
\end{array}\right\} \\
& =\overbrace{\left\{\begin{array}{lllllllllll}
1 & 2 & \cdots & i & \cdots & n-1 & n
\end{array}\right\} \oplus\left\{\begin{array}{llllllll}
1 & 2 & \cdots & i & \cdots & n-1 & n
\end{array}\right\}}^{\text {only the multiplication of the last term,i.e.without any reiteration }}
\end{aligned}
$$


Two penalties are paid for the rectification of the order of the second sequence: 1) replacing the sign () with: $\oplus ; 2)$ the sequence in the reverse order is visually rectified but the operations are carried out as in (4.11.d). Although this is counterintuitive, the algorithm is robust in both programming and for humans. The outcome is the creation of a clear identity for regeneration, which is underpinned by appropriate notations.

\subsection{Recursive Products}

The operators presented above can be modified to cope with recursive sequences, in which the operations apply on the trailing terms, such as Fibonacci sequences but they are not introduced in this paper.

\subsection{Commutative, Associative and Distributive Laws}

Conducemental algebra has its own commutative, associative and distributive laws, as follows.

The commutative law (the ability to swap):

$$
\begin{aligned}
& \{S\}_{1} \circ\{S\}_{2}=\{S\}_{2} \circ\{S\}_{1} \\
& \{S\}_{1} \odot\{S\}_{2}=\{S\}_{2} \odot\{S\}_{1} \\
& \{S\}_{1} \oplus\{S\}_{2}=\{S\}_{2} \oplus\{S\}_{1} \\
& \{S\}_{1} \hat{\oplus}\{S\}_{2}=\{S\}_{2} \hat{\oplus}\{S\}_{1}
\end{aligned}
$$

The associative law (grouping of sequences together):

$$
\begin{gathered}
\{S\}_{1} \circ\{S\}_{2} \circ\{S\}_{3}=\{S\}_{3} \circ\{S\}_{2} \circ\{S\}_{1}=\{S\}_{1} \circ\{S\}_{3} \circ\{S\}_{2} \\
\{S\}_{1} \odot\{S\}_{2} \odot\{S\}_{3}=\{S\}_{3} \odot\{S\}_{2} \odot\{S\}_{1}=\{S\}_{1} \odot\{S\}_{3} \odot\{S\}_{2} \\
\{S\}_{1} \oplus\{S\}_{2} \oplus\{S\}_{3}=\{S\}_{3} \oplus\{S\}_{2} \oplus\{S\}_{1}=\{S\}_{1} \oplus\{S\}_{3} \oplus\{S\}_{2} \\
\{S\}_{1} \hat{\oplus}\{S\}_{2} \hat{\oplus}\{S\}_{3}=\{S\}_{3} \hat{\oplus}\{S\}_{2} \hat{\oplus}\{S\}_{1}=\{S\}_{1} \hat{\oplus}\{S\}_{3} \hat{\oplus}\{S\}_{2}
\end{gathered}
$$

The distributive law does not hold for the simultaneous direct and regenerating/convoluting products:

$$
\left(\{S\}_{1} \circ / \odot\{S\}_{2}\right) \oplus / \hat{\oplus}\{S\}_{3} \neq\{S\}_{1} \circ / \odot\left(\{S\}_{2} \oplus / \hat{\oplus}\{S\}_{1}\right)
$$

where $\circ / \odot$ or $\oplus / \hat{\oplus}$ represent one or the other multiplication rule.

\section{Operators for Reducement-Inverse Problems}

\section{Statement of the Problem}

In conventional algebra, inverse problems are often ill-posed, when one or more of the three conditions outlined in Section 4.1 are not satisfied. This paper proposes to refer to the inverse problem of arithmetic calculus as the reducement problem. This section shows that reducements involve the solution of a system of equations, which are properly-posed and can be solved by a "single sweep" method as the equations in each sweep are a chain of a single linear equation with one unknown. So the reducement problem is slightly more complex than the conducement problem but this can be reduced to algorithm. Notably, the reducement problem unearths the structure in number sequences, which are encapsulated by conducement operations.

Operators are needed to implement inverse problems in arithmetic calculus. Consider the example of the product of two sequences processed by conducemental algebra:

$$
\left\{\begin{array}{lll}
1 & 2 & 1
\end{array}\right\} \hat{\oplus}\left\{\begin{array}{llll}
1 & 3 & 3 & 1
\end{array}\right\}=\left\{\begin{array}{llllll}
1 & 5 & 10 & 10 & 5 & 1
\end{array}\right\}
$$

Consider the $3^{\text {rd }}$ term worked out as: $1 \times 3+2 \times 3+1 \times 1=10$. The value " 10 " encapsulates the conducement operation and this makes it unthinkable to reverse the above operation. The objective is now to find a systematic way of carrying out the following operations:

$$
\left\{\begin{array}{llllll}
1 & 5 & 10 & 10 & 5 & 1
\end{array}\right\} \text { reduces to: }\left\{\begin{array}{llll}
1 & 3 & 3 & 1
\end{array}\right\} \text { and }\left\{\begin{array}{lll}
1 & 2 & 1
\end{array}\right\}
$$


$\left\{\begin{array}{llllll}1 & 5 & 10 & 10 & 5 & 1\end{array}\right\} /\left\{\begin{array}{lll}1 & 2 & 1\end{array}\right\}$ to work out: $\left\{\begin{array}{llll}1 & 3 & 3 & 1\end{array}\right\} ;$

and

$$
\left\{\begin{array}{llllll}
1 & 5 & 10 & 10 & 5 & 1
\end{array}\right\} /\left\{\begin{array}{llll}
1 & 3 & 3 & 1
\end{array}\right\} \text { to work out: }\left\{\begin{array}{lll}
1 & 2 & 1
\end{array}\right\} .
$$

In the above, it is proposed to replace "/” with “ $\oslash$ ”. The key for the solution of the above problems is the inverse problem and this is solved by the following theorem:

Theorem 5.1: The inverse of any sequence: $\left\{\begin{array}{lllll}t_{1} & t_{2} & t_{3} & t_{4} & \cdots\end{array}\right\}$, is given by $\left\{\begin{array}{lllll}i_{1} & i_{2} & i_{3} & i_{4} & \cdots\end{array}\right\}$, the values of which are:

$$
\left\{\begin{array}{llll}
t_{1} & t_{2} & t_{3} & t_{4}
\end{array}\right\} \hat{\oplus}\left\{\begin{array}{llll}
i_{1} & i_{2} & i_{3} & i_{4}
\end{array}\right\}=\left\{\begin{array}{llll}
1 & 0 & 0 & 0
\end{array}\right\}
$$

where,

$$
i_{1} \Rightarrow i_{1} * t_{1}=1 ; \Rightarrow i_{1}=1 / t_{1}
$$

and

$$
\text { for } i_{2} \Rightarrow t_{1} * r_{2}+t_{2} * r_{1}=0 \text {; where } i_{1} \text { is already solved in (5.2.b)) }
$$

and for $i_{3} \Rightarrow t_{1} * r_{3}+t_{2} * r_{2}+t_{3} * r_{1}=0$; where $i_{1}$ is solved in (5.2.b) and $i_{2}$ is solved (5.2.c)).

Remark 5.1: These equations form a simple system of linear equations chained to one another, which are solved in a single sweep by finding the value of one and feeding it to the next. The solutions are exact but the inverse sequence is open for a closed sequence and vice versa (closed sequence for an open sequence), see below:

$$
\begin{aligned}
& 1 /\{1 \quad 1\}=\left\{\begin{array}{lllll}
1 & -1 & 1 & -1 & \cdots
\end{array}\right\} \\
& 1 /\left\{\begin{array}{lll}
1 & 2 & 1
\end{array}\right\}=1 \oslash\left\{\begin{array}{lll}
1 & 2 & 1
\end{array}\right\}=\left\{\begin{array}{lllll}
1 & -2 & 3 & -4 & \cdots
\end{array}\right\} \\
& 1 /\left\{\begin{array}{llll}
1 & 3 & 3 & 1
\end{array}\right\}=1 \oslash\left\{\begin{array}{llll}
1 & 3 & 3 & 1
\end{array}\right\}=\left\{\begin{array}{lllll}
1 & -3 & 6 & -10 & \cdots
\end{array}\right\} \\
& 1 /\{1 \quad-1\}=\left\{\begin{array}{lllll}
1 & 1 & 1 & 1 & \cdots
\end{array}\right\} \\
& 1 /\left\{\begin{array}{lll}
1 & -2 & 1
\end{array}\right\}=1 \oslash\left\{\begin{array}{lll}
1 & -2 & 1
\end{array}\right\}=\left\{\begin{array}{lllll}
1 & 2 & 3 & 4 & \cdots
\end{array}\right\} \\
& 1 /\left\{\begin{array}{llll}
1 & -3 & 3 & -1
\end{array}\right\}=1 \oslash\left\{\begin{array}{llll}
1 & -3 & 3 & -1
\end{array}\right\}=\left\{\begin{array}{lllll}
1 & 3 & 6 & 10 & \cdots
\end{array}\right\} \\
& 1 /\left\{\begin{array}{lllll}
1 & 1 & 1 & 1 & \cdots
\end{array}\right\}=\left\{\begin{array}{ll}
1 & -1
\end{array}\right\} \\
& 1 /\left\{\begin{array}{lllll}
1 & 2 & 3 & 4 & \cdots
\end{array}\right\}=1 \oslash\left\{\begin{array}{lllll}
1 & 2 & 3 & 4 & \cdots
\end{array}\right\}=\left\{\begin{array}{lll}
1 & -2 & 1
\end{array}\right\} \\
& 1 /\left\{\begin{array}{llllll}
1 & 3 & 6 & 10 & \cdots
\end{array}\right\}=1 \oslash\left\{\begin{array}{llllll}
1 & 3 & 6 & 10 & \cdots
\end{array}\right\}=\left\{\begin{array}{llll}
1 & -3 & 3 & -1
\end{array}\right\} \\
& 1 /\left\{\begin{array}{lllll}
1 & -1 & 1 & -1 & \cdots
\end{array}\right\}=\left\{\begin{array}{ll}
1 & 1
\end{array}\right\} \\
& 1 /\left\{\begin{array}{lllll}
1 & -2 & 3 & -4 & \cdots
\end{array}\right\}=1 \oslash\left\{\begin{array}{lllll}
1 & -2 & 3 & -4 & \cdots
\end{array}\right\}=\left\{\begin{array}{lll}
1 & 2 & 1
\end{array}\right\} \\
& 1 /\left\{\begin{array}{lllll}
1 & -3 & 6 & -10 & \cdots
\end{array}\right\}=1 \oslash\left\{\begin{array}{lllll}
1 & -3 & 6 & -10 & \cdots
\end{array}\right\}=\left\{\begin{array}{llll}
1 & 3 & 3 & 1
\end{array}\right\}
\end{aligned}
$$

The above are textbook stuff but the point is that they can be processed in a systemic way by Theorem 5.1, which itself is a statement of the obvious for mathematicians but the knowledge is yet to be transformed into working tools. The ground for this transformation is prepared by the following.

Corollary 5.1: Any conducemental sequence, which is the product of two sequences, can be factorised into the product of two sequences by multiplying it with the inverse of any one of its constituent sequences, as in the example below:

$$
\begin{aligned}
& \left\{\begin{array}{llllll}
1 & 5 & 10 & 10 & 5 & 1
\end{array}\right\} \oslash\left\{\begin{array}{llll}
1 & 3 & 3 & 1
\end{array}\right\} \\
& =\left\{\begin{array}{llllllllll}
1 & 5 & 10 & 10 & 5 & 1
\end{array}\right\} \oplus\left\{\begin{array}{llllllll}
1 & -3 & 6 & -10 & 15 & -21
\end{array}\right\}=\left\{\begin{array}{llllll}
1 & 2 & 1 & 0 & 0 & 0
\end{array}\right\}
\end{aligned}
$$


The above is just one possibility, but the factorisation can be carried out systematically by dividing it to: $\{0\}$, $\left\{\begin{array}{ll}1 & 1\end{array}\right\},\left\{\begin{array}{lll}1 & 2 & 1\end{array}\right\},\left\{\begin{array}{llll}1 & 3 & 3 & 1\end{array}\right\},\left\{\begin{array}{lllll}1 & 4 & 6 & 4 & 1\end{array}\right\},\left\{\begin{array}{llllll}1 & 5 & 10 & 10 & 5 & 1\end{array}\right\}$.

This corollary seems to lead to a trivial outcome, as the constituent sequences of the product must be known in advance. However, normally the factorisation does not lead to a single outcome but the identification of all the possible outcomes. So factorisation is a program to start with one possible sequence; to keep increasing its order until the identification of all possible constituent sequences; and to select the meaningful ones.

Corollary 5.2: The deconvolution problem is properly-posed through the conducemental algebra but improperly-posed through conventional algebra. Consider the problem (5.6):

$$
\left\{\begin{array}{lll}
1 & 2 & 1
\end{array}\right\} \hat{\oplus}\left\{\begin{array}{llll}
x_{1} & x_{2} & x_{3} & x_{4}
\end{array}\right\}=\left\{\begin{array}{llllll}
1 & 5 & 10 & 10 & 5 & 1
\end{array}\right\}
$$

Literally, the above leads to 6 equations with four unknowns, which renders the problem as ill-posed. This does not arise in reformulating (5.6) into the format by conducemental algebra:

$$
\left\{\begin{array}{llllll}
1 & 5 & 10 & 10 & 5 & 1
\end{array}\right\} \oslash\left\{\begin{array}{lll}
1 & 2 & 1
\end{array}\right\}=\left\{\begin{array}{lllllll}
1 & 5 & 10 & 10 & 5 & 1
\end{array}\right\} \oplus\left\{\begin{array}{lllll}
1 & -2 & 3 & -4 & \cdots
\end{array}\right\}
$$

Based on conventional algebra, there are 6 equations but 4 unknowns and therefore the problem in principle is improperly-posed; whereas this issue is completely irrelevant in conducemental algebra:

$$
\begin{aligned}
& \left\{\begin{array}{llllll}
1 & 5 & 10 & 10 & 5 & 1
\end{array}\right\} \oslash\left\{\begin{array}{llll}
1 & 3 & 3 & 1
\end{array}\right\} \\
& =\left\{\begin{array}{llllllll}
1 & 5 & 10 & 10 & 5 & 1
\end{array}\right\} \oplus\left\{\begin{array}{llllllll}
1 & -3 & 6 & -10 & 15 & -21
\end{array}\right\}=\left\{\begin{array}{llllll}
1 & 2 & 1 & 0 & 0 & 0
\end{array}\right\}
\end{aligned}
$$

To understand the above better, consider the reformulation of (5.5), as follows:

$$
\left\{\begin{array}{llllll}
1 & 2 & 1 & 0 & 0 & 0
\end{array}\right\} \oplus\left\{\begin{array}{llllll}
x_{1} & x_{2} & x_{3} & x_{4} & x_{5} & x_{6}
\end{array}\right\}=\left\{\begin{array}{llllll}
1 & 5 & 10 & 10 & 5 & 1
\end{array}\right\}
$$

The above transforms the convolution product into the regeneration product and there are literally, 6 equations and 6 unknowns. However, when the above is subject to measurement errors, the problem becomes properlyposed but ill-conditioned but the strategies to deal with ill-conditioned problems of this nature are not the focus of this paper.

\section{The Problem of Conducement-Applications}

Conducement is a proposed term for multiplying the corresponding elements of two sequences by using any variation of the direct and/or convolution product rules, which intrinsically involve integration. It gives rise to a series of elements, within which the preceding elements are nested in as sub-terms but encapsulated. Summing the respective sub-terms gives the value of each and all of the elements but the inherent structures are lost.

\subsection{Regenerating Natural Numbers}

Arithmetic calculus sheds a new light to sequences of natural numbers by penetrating into their very basics. The sequence of natural numbers can be constructed without any assumption. This involves priming in the first place from a kernel, where the kernel is $\langle 1\rangle$ for natural numbers. The concept of priming is detailed by the author [2] in the context of generalising theory of evolutionary to explain the transformation of an object into different uses. For instance, arithmetic calculus primes the kernel by multiplying the kernel with the sequence of counter, represented by: $\{\overline{1}\}$, as follows.

Step 1: Generation 0: Priming Generation 0 from $\langle 1\rangle$ and $\{\overline{1}\}$ :

$$
\langle 1\rangle \circ\{\overline{1}\}=\langle 1\rangle \oplus\{\overline{1}\}=\langle 1\rangle \otimes\{\overline{1}\}=\{1\}=\underbrace{\left\{\begin{array}{c}
\mathbb{N} \\
G=0
\end{array}\right.}_{\varphi=0}\}^{D=0}
$$

Kernel: $\langle 1\rangle$ is not yet a sequence but is generically referred to as building blocks; $\{\overline{1}\}$ is a rule and its overbar discerns its value from any other value; $\{1\}$ is an element of a sequence (i.e. a countable number). Its specification is:

Product Specification: Kernel $=\langle 1\rangle ;$ Generation $=0 ;$ Degree $=0$; Order $=0$. 
Step 2: Generation 1: Generation 1 of natural numbers is regenerated from (6.1.a), as follows:

$$
\{1\} \oplus\left\{\begin{array}{ccccc}
1 & 1 & 1 & 1 & \cdots \\
0 & 0 & 0 & 0 & 0
\end{array}\right\}=\left\{\begin{array}{lllll}
1 & 1 & 1 & 1 & \cdots
\end{array}\right\}=\underbrace{\left\{\begin{array}{c}
\mathbb{N} \\
G=1
\end{array}\right.}_{\varphi=0}\}^{D=0}
$$

where the specific form of the sequence of counters signifies that in this operation, there is no operation of addition. The inverse of (6.1.a) is well known and is: $\left\{\begin{array}{ll}1 & -1\end{array}\right\}$. (6.1.b) has the following Specification:

Product Specification: Kernel $=\langle 1\rangle ;$ Generation $=1 ;$ Degree $=0 ;$ Order $=0$.

Step 3: Generation 2: The sequence of natural numbers is regenerated, as:

$$
\left\{\begin{array}{llll}
1 & 1 & 1 & \cdots
\end{array}\right\} \oplus\left\{\begin{array}{llll}
1 & 1 & 1 & \cdots
\end{array}\right\}=\left\{\begin{array}{lll}
\underbrace{1^{\mathrm{st}} \text { eerm }}_{1 \times 1} & \frac{2^{\text {nd }} \text { term }}{1 \times 1+1 \times 1} & \cdots \\
1 & \cdots
\end{array}\right\}=\left\{\begin{array}{llll}
1 & 2 & 3 & \cdots
\end{array}\right\}=\underbrace{\{\underbrace{\mathbb{N}}_{G=2}}_{\varphi=1}\}^{D=0}
$$

The inverse of (6.1.c) is well known from algebra and is: $\left\{\begin{array}{lll}1 & -2 & 1\end{array}\right\}$. The Specification for (6.1.c) is:

$$
\text { Product Specification: } \text { Kernel }=\langle 1\rangle ; \text { Generation }=2 ; \text { Degree }=0 ; \text { Order }=1 \text {. }
$$

Remark 6.1: (6.1.c) is a special sequence for being natural numbers. It is derived through (6.1.a)-(6.1.c) from the first principles by priming its Kernel: $\langle 1\rangle$ and the most basic rule expressed by the unitary sequence of counters. Arithmetic calculus unearths the role of selecting the rule in conducing "2" denoted by $\left(\frac{2^{\text {nd }} \text { term }}{1 \times 1+1 \times 1}\right)$, and any other term. Selection is unlike assumption, as the assumption should be refined but selection need not. Thus, with arithmetic calculus the proof of $1+1=2$ becomes irrelevant. Moreover, each rule leads to a product with a potential for selection. The mathematical symbol of "2" and it natural language name in any language (and any other numbers) are by pure convention driven by their selective advantage. Of course, these artefacts were selected in the cultural life first and their derivation is just a mathematical strive at a much later stage. The paper promotes a new thinking based on rule's operated on building blocks. This questions the wisdom of proving that $1+1=2$, as in the legend of Bertrand Russell and Alfred North Whitehead who spent some 40 pages in their Principia Mathematica in the early $20^{\text {th }}$ century to state that "Therefore, $1+1=2$."

Step 4: Generation $g$ : The Order $g$ sequence may be regenerated as following:

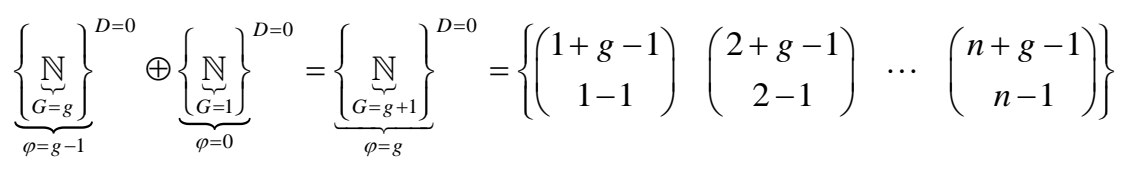

Product Specification: Kernel $=\langle 1\rangle$; Generation $=g+1$; Degree $=0$; Order $=g$.

Remark 6.2: The author is not claiming originality for the above age old sequences but their regeneration in the fashion of arithmetic calculus underpins a fresh view and underpins a good reason for their notations, where existing ones fail to reflect the complexity of the problems.

\subsection{Multinomial Sequences: Recursive or Convoluting}

The concept of order/generation/degree/kernel, as well as the above multiplication rules are general and can equally be applied to binomial, trinomial or multinomial sequences. These are not presented here, except the following examples: 
Trinomial:

$$
\left\{\begin{array}{llll}
1 & 1 & 1 & 1
\end{array}\right\} \hat{\oplus}\left\{\begin{array}{lll}
1 & 1 & 1
\end{array}\right\}=\left\{\begin{array}{llllll}
1 & 2 & 3 & 3 & 2 & 1
\end{array}\right\}
$$

Multinomial:

$$
\left\{\begin{array}{llllll}
1 & 2 & 3 & 3 & 2 & 1
\end{array}\right\} \hat{\oplus}\left\{\begin{array}{llll}
1 & 1 & 1 & 1
\end{array}\right\}=\left\{\begin{array}{lllllllll}
1 & 3 & 6 & 9 & 10 & 9 & 6 & 3 & 1
\end{array}\right\}
$$

Remark 6.3: The inverses for the above are not given here but their reducementals are all systematic.

\section{Factorisation by Conducemental Algebra-Heuristic Rules and Applications}

\subsection{Factorisation of Sequences}

Conducemental algebra shows that after conducing two sequences, any trace of the multiplication operations are encapsulated. However, an expression of these operations is a structure that can be unearthed by inverse problems or by factorisation. The idea is not to reverse the operation to find the original sequences used in the multiplication but to find the structure hidden in the product. This structure is expressed by thinking in terms of building blocks and rule and this in turn is consolidated in terms of reducementals. Complex conducemental sequences (the parent sequence) encapsulate in themselves the information of their structures but this can be unearthed. Notably, reducementals describe each element but not the whole parent sequence. In this method the sequence of counters is assumed beforehand but the problem is to find the values of sequence of invariants, where their product determines the given sequence.

The author has a devised a number of techniques to derive the reducementals but this paper presents two of them: the age-old method of differences capable of depicting a graphical image of underlying ideas and the conducemental algebra with appropriate operators to derive the operations in a systematic way. The sequence of counters is conveniently based on the binomial expression, serving as an operator:

$$
\begin{aligned}
& \left\{\left(\begin{array}{l}
n \\
n
\end{array}\right)\left(\begin{array}{c}
n \\
n-1
\end{array}\right)\left(\begin{array}{c}
n \\
n-2
\end{array}\right)\left(\begin{array}{c}
n \\
n-3
\end{array}\right) \cdots\right\} \\
& \left\{\left(\begin{array}{c}
n \\
n-1
\end{array}\right)\left(\begin{array}{c}
n \\
n-2
\end{array}\right)\left(\begin{array}{c}
n \\
n-3
\end{array}\right)\left(\begin{array}{c}
n \\
n-4
\end{array}\right) \cdots\right\} \\
& \left\{\left(\begin{array}{c}
n+m-1 \\
n-1
\end{array}\right)\left(\begin{array}{c}
n+m-2 \\
n-2
\end{array}\right)\left(\begin{array}{c}
n+m-3 \\
n-3
\end{array}\right)\left(\begin{array}{c}
n+m-4 \\
n-4
\end{array}\right) \quad \cdots\right\}
\end{aligned}
$$

In fact the first two operators are the binomial terms, in which (7.1.a) commences from: $\left(\begin{array}{l}n \\ n\end{array}\right)$ but (7.1.b) is curtailed and commences from: $\left(\begin{array}{c}n \\ n-1\end{array}\right)$, where these operators describe the rule of object selection without replacement; whereas, $\left(\begin{array}{c}n+m-1 \\ n-1\end{array}\right)$ represents object selection with replacement. Using any of the above operators, even the well known power sequence can be factorised as:

$$
\left\{\mathcal{R} \mid\left\{\begin{array}{llll}
1^{m} & 2^{m} & \cdots & n^{m}
\end{array}\right\}\right\}={ }^{\text {Red }}\{\mathbb{N}\}^{m}=\left\{\begin{array}{llll}
\chi_{1} & \chi_{2} & \cdots & \chi_{m}
\end{array}\right\} \odot\left\{\left(\begin{array}{c}
n \\
n-1
\end{array}\right)\left(\begin{array}{c}
n \\
n-2
\end{array}\right) \cdots\left(\begin{array}{c}
n \\
n-m
\end{array}\right)\right\}
$$

where, $\chi \quad$ values are calculated by conducemental algebra.

\subsection{Method of Difference}

Traditional method of differences is explicitly blind to the sequence of counters but the example presented below shows the way this method can be brought to life when it is associated with the sequence of counters. Nonetheless, the method of differences fails to elucidate a structure that is hidden in each and all of the elements of the parent sequence; whereas conducemental algebra thrives on unravelling this structure. This is illustrated 
through the following example.

Example 7.1: Consider the example of:

$$
\{\underbrace{\{\underbrace{\mathbb{N}}_{G=2}\}^{2}}_{\varphi=1} \oplus \underbrace{\left\{\begin{array}{c}
\mathbb{N} \\
G=3
\end{array}\right\}}_{\phi=1}\} \circ \underbrace{\left\{\begin{array}{c}
\mathbb{N} \\
G=2
\end{array}\right\}}_{\varphi=1}=\left\{\begin{array}{lllllllllll}
1 & 14 & 81 & 308 & 910 & 2268 & 4998 & 10032 & 18711 & 32890 & \cdots
\end{array}\right\}
$$

The sequence is remarkably complex but the well-known method of differences is remarkably straightforward. Table 1 presents this method in its traditional form, which comprises elements in each row, as follows:

Row 2: presents the values of the parent sequence expressed by (7.3).

Rows 2-9: present the application of the difference method. The elements in each row are calculated as the difference between the two elements in its immediate upper row, one on the top and the other prior to it. This method is only applicable when the sequence is generated by the sequence of natural numbers but breaks down when applied to operations on different sequences (e.g. Euler Numbers or Pascal Triangle). Nothing much can be said of the table. The conducemental algebra makes this table redundant but uses it for a graphic display of the problem, as below.

The aim here is to upgrade explicitly the capability of the table of differences, even though the conducemental algebra makes it redundant. As shown in Table 1, the aim is achieved by: 1 ) introducing the sequence of counters to Row 1 and Column 1;2) this sequence is associated with the diagonals of the table; 3) the association is by the direct multiplication rule. It is proposed to refer to the products as reducementals, which represent each and every element of the parent sequence. Systematic variations in the sequence of counters are striking but this isless obvious in the values of the elements of each row and diagonal and the key is to unearth these variations. These are captured below in qualitative terms but their quantitative account is presented in the next sections. The specification of (7.3) is as follows:

Generation: $G=g=4$; Degree: $D=d=3$; Order: degree + generation $-1=\varphi=\Phi=4+3-1=6$.

Kernel: Kernel size $=3$; Kernel: $\left\langle\begin{array}{lll}1 & 7 & 4\end{array}\right\rangle$; Maximum size of sequence of invariant: $\varphi+1=6+1=7$.

Admittedly, the rules appropriate for the above specification are not detailed to manage the paper's length.

Remark 7.1: Each row in the table forms a complex open conducemental sequence. It is proposed to refer to: 1) the highest row as the parent sequence (Order, $\varphi=\Phi+1)$; 2 ) lowest rows as the kernel ( $\varphi=0)$, which is no longer a sequence; 3 ) intermediate rows as a sequence with specific order number (order, $\varphi=\varnothing$ ). The sequence with specific orders, $\varnothing$, is related to its upper row of order: $\varnothing+1$ (by multiplying it by: $\oplus\{1-1\}$ ). All these sequences are interoperable and this includes any sequences not commencing from its first element. The sequences commencing from the first element are to be referred to as leading sequences but conducemental algebra is powerful for coping with the full description of any trailing sequence, which skips any given number of elements from its first element.

Remark 7.2: The diagonals express the sequences of invariants, which are closed conducemental sequences. Each diagonal is associated with a specific sequence of counter using the direct product rule to be referred to as a reducemental.

Remark 7.3: The sequences of invariants (i.e. the values in each diagonal) are interoperable with each other but the degree and generation of the parent sequence plays a pivotal role. The paper delineates five possible diagonals causing transitions towards generic behaviours, as follows:

Transitional Diagonal 4 (TD4): This refers to the kernel, which is the end-product of transforming the diagonals into a row. A parent sequence not rendering a kernel is not conducemental.

Transitional Diagonal 3 (TD3): This refers to diagonals bearing the effects of direct products encapsulated by the parent sequences. The diagonals in between TD4 and TD3 include a sequence of counter which is hybrid of (7.1.b), (7.1.c).

Transitional Diagonal 2 (TD2): This refers to diagonals bearing the effects of regeneration products in the parent sequences. The diagonals in the range of TD3 and TD2 are associated with (7.1.b).

Transitional Diagonal 1 (TD1): This employs (7.1.a) for its sequence of counters and has the largest size.

Transitional Diagonal 0 (TD0): The diagonals associated with TD0 are any trailing sequences. 


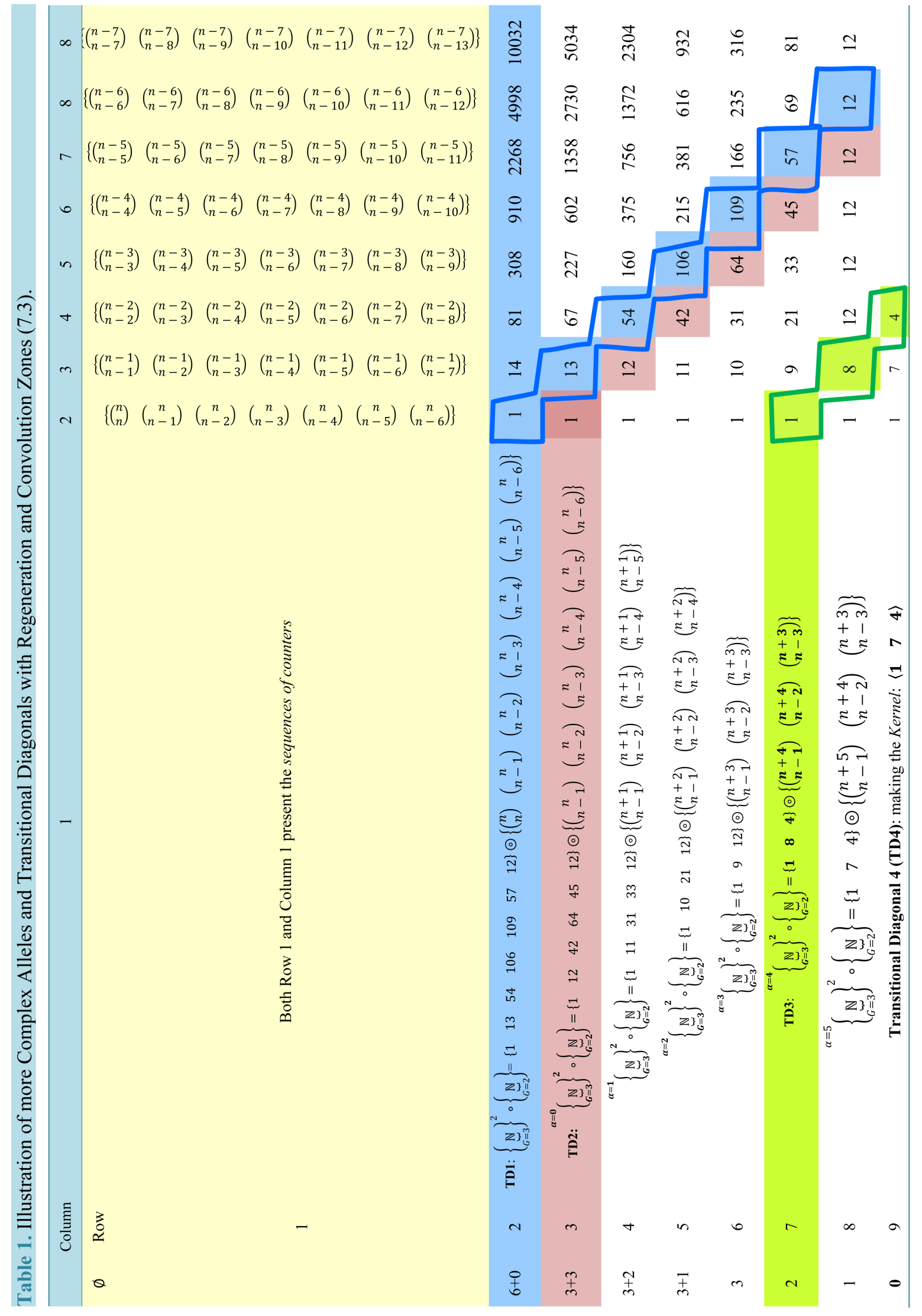


Remark 7.4: To the right of TD0, the sequence in each row is always a trailing one and therefore its starting element is never the first element. To the left of TD0, the sequence in each row is a leading one and starts with the first element of the parent sequence.

Remark 7.5: The reducementals within TD1 and TD4 are interoperable expressions of one another for the parent sequence and may be referred to as alleles.

Remark 7.6: The sequences of invariants in the range of TD4-TD3 have a constant number of elements and this signifies that their interoperability transformers employ the regeneration product rule (these will be clear in the next section).The fact underlying this statement is underpinned by order, degree and generation.

Remark 7.7: The number of elements in sequences of invariants in the range from TD3 to TD2 increases (or decreases in the opposite direction) by " 1 " and this signifies that their interoperability transformers employ the convolution product rule. The fact underlying this statement is underpinned by order, degree and generation.

\section{Reducement and Interoperability-Heuristic Rules and Applications}

This section introduces heuristically an alternative reducement approach to conducemental sequences using examples through a set of rules.

\subsection{Interoperability between Higher and Lower Order Conducemental Sequences}

Rule 8.1: A complex conducemental sequence of natural numbers of Order $=\phi$ is reducible to a lower order of $\varphi$ using the direct product rule by multiplying the sequence with the following inverse sequence:

$$
\left\{\left(\begin{array}{c}
\phi-\varphi \\
0
\end{array}\right)(-1)^{1}\left(\begin{array}{c}
\phi-\varphi \\
1
\end{array}\right) \quad(-1)^{2}\left(\begin{array}{c}
\phi-\varphi \\
2
\end{array}\right) \cdots(-1)^{i}\left(\begin{array}{c}
\phi-\varphi \\
i
\end{array}\right)(-1)^{\phi-\varphi}\left(\begin{array}{c}
\phi-\varphi \\
\phi-\varphi
\end{array}\right)\right\}
$$

Example 8.1: Using Example 7.1, the inverse reducement sequences from $\phi=6$ to $\phi=1$ are: For $\phi-\varphi=1$, the inverse sequence is:

$$
\overbrace{\{1-1\}}^{\phi-\varphi=1}
$$

For $\phi-\varphi=2$, the inverse sequence is:

$$
\overbrace{\{1-2 \quad 1\}, \cdots}^{\phi-\varphi=2}
$$

For $\phi-\varphi=6$, the inverse sequence is:

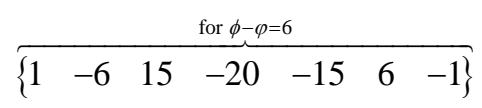

Note that for $\phi-\varphi>1,2, \cdots, 6$ in the above examples, the particular terms becomes zero (e.g. $\left(\begin{array}{l}6 \\ 7\end{array}\right)=0$ ). To reduce to the order of the kernel, the order has to be increased by " 1 " and hence in Example 7.1:

$$
\begin{aligned}
\left\{\begin{array}{lllllll}
1 & 14 & 81 & 308 & 910 & 2268 & \cdots
\end{array}\right\} \oplus\left\{\begin{array}{llllllll}
1 & -7 & 21 & -35 & 35 & -21 & 7 & 1
\end{array}\right\} \\
=\left\{\begin{array}{llllll}
1 & 7 & 4 & 0 & \cdots
\end{array}\right.
\end{aligned}
$$

Alternatively, any of the sequence of order $\varphi$ may be regenerated from the kernel $\left\langle\begin{array}{llll}1 & 7 & 4\end{array}\right\rangle$ but the kernel first has to be primed to obtain: $\left\{\begin{array}{lll}1 & 7 & 4\end{array}\right\}$.

\subsection{Sequences of Invariants Obtained Directly from Parent Sequences}

The reducement rule for obtaining the values of sequences of invariants is equally simple and flexible and any of the alleles are obtained by simply regenerating the parent sequence using inverses of natural number of increasing orders. 
Sequence of Invariants: Work out the diagonals (sequence of invariants) in Table 1.

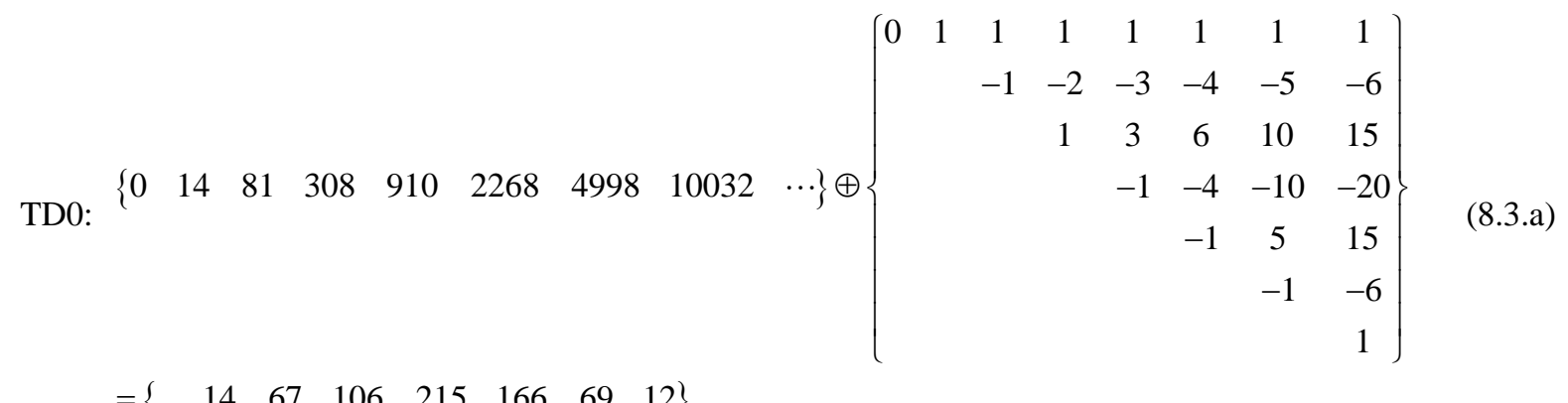

$$
\begin{aligned}
& =\left\{\begin{array}{lllllll}
14 & 67 & 106 & 215 & 166 & 69 & 12
\end{array}\right\}
\end{aligned}
$$

TD1:

$$
\left\{\begin{array}{lllllll}
1 & 14 & 81 & 308 & 910 & 2268 & \cdots
\end{array}\right\} \oplus\left\{\begin{array}{cccccc}
1 & 1 & 1 & 1 & 1 & 1 \\
& -1 & -2 & -3 & -4 & -5 \\
& & 1 & 3 & 6 & 10 \\
& & -1 & -4 & -10 \\
& & & 1 & 5 \\
& & & & & \\
& & & & &
\end{array}\right\}=\left\{\begin{array}{llllllll}
1 & 13 & 54 & 106 & 109 & 57 & 12
\end{array}\right\}
$$

TD2:

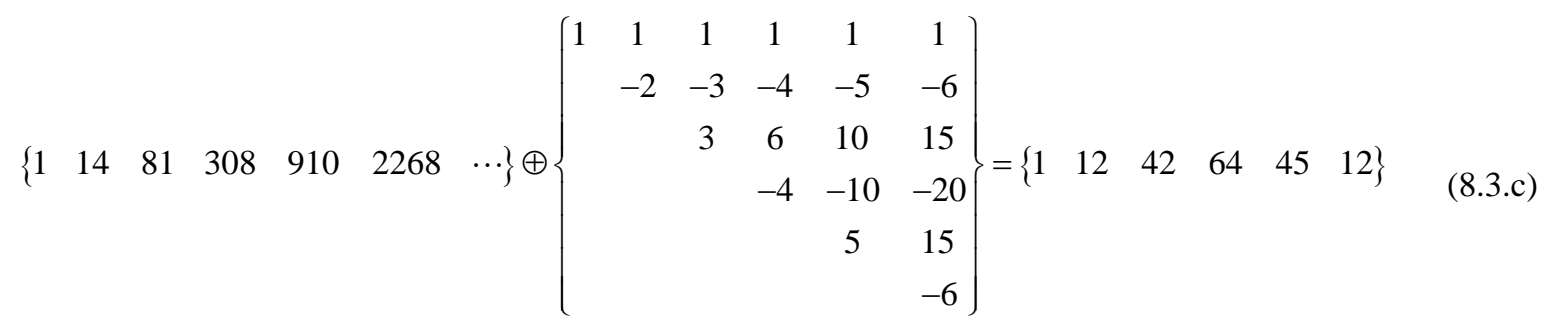

TD2-TD3.1:

$$
\left\{\begin{array}{lllllll}
1 & 14 & 81 & 308 & 910 & 2268 & \cdots
\end{array}\right\} \oplus\left\{\begin{array}{ccccc}
1 & 1 & 1 & 1 & 1 \\
& -3 & -4 & -5 & -6 \\
& 6 & 10 & 15 \\
& & -10 & -20 \\
& & & & 15
\end{array}\right\}=\left\{\begin{array}{ccccc}
1 & 11 & 31 & 33 & 12
\end{array}\right\}
$$

TD2-TD3.2:

$$
\left\{\begin{array}{lllllll}
1 & 14 & 81 & 308 & 910 & 2268 & \cdots
\end{array}\right\} \oplus\left\{\begin{array}{cccc}
1 & 1 & 1 & 1 \\
-4 & -5 & -6 \\
& 10 & 15 \\
& & -20
\end{array}\right\}=\left\{\begin{array}{llll}
1 & 10 & 21 & 12
\end{array}\right\}
$$

TD2-TD3.3: 


$$
\left\{\begin{array}{lllllll}
1 & 14 & 81 & 308 & 910 & 2268 & \ldots
\end{array}\right\} \oplus\left\{\begin{array}{ccc}
1 & 1 & 1 \\
-5 & -6 \\
& & 15
\end{array}\right\}=\left\{\begin{array}{lll}
1 & 9 & 12
\end{array}\right\}
$$

TD3-TD4.1:

$$
\left\{\begin{array}{lllllll}
1 & 14 & 81 & 308 & 910 & 2268 & \cdots
\end{array}\right\} \oplus\left\{\begin{array}{cccc}
1 & 1 & 1 & 1 \\
-6 & -7 & -7 \\
& 21 & 21 \\
& & -35
\end{array}\right\}=\left\{\begin{array}{lllll}
1 & 8 & 4 & 0 & \cdots
\end{array}\right\}
$$

TD3-TD4.2:

$$
\begin{gathered}
\left\{\begin{array}{ccccccc}
1 & 14 & 81 & 308 & 910 & 2268 & \cdots
\end{array}\right\} \oplus\left\{\begin{array}{cccccc}
1 & 1 & 1 & 1 & 1 & 1 \\
-7 & -7 & -7 & -7 & -7 \\
& 21 & 21 & 21 & 21 \\
& & -35 & -35 & -35 \\
& & & 35 & 35 \\
& & & & & -21
\end{array}\right\}=\left\{\begin{array}{llllll}
1 & 7 & 4 & 0 & 0 & 0
\end{array}\right\} \\
\operatorname{TD} 1:\left\langle\begin{array}{lllll}
1 & 7 & 4
\end{array}\right\rangle
\end{gathered}
$$

It is noted that in both (8.2.a) and (8.2.b), the size of the diagonals is $7=\varphi+1=6+1$. For (8.2.c) to (8.2.f), the size reduced by " 1 " for each diagonal until the size becomes 3 and from here until the derivation of the kernels, it remains a constant value of 3 . These are explainable by the virtue of degree, generation and order, although the presentation of the full rules for these dimensional parameters is beyond the remit of this paper.

Remark 8.1: The inverse sequences used in (8.3.a)-(8.3.h) are generic and not specific to Example 7.1. The dimensional parameters in the above are the degree as in (TD3-TD4) and generation as in (TD2-TD3) with for any other parent conducemental sequences calculated by appropriate rules to be presented by the author in the near future.

Remark 8.2: Further remarks on transitional diagonals are:

TD0: is open-ended to the right hand side of Table 1 but the starting element is the key to identify the sequence. Inverse sequences for TD0 are identical and the changes in the sequence of invariants are systematic.

TD1: This diagonal, associated with (7.1.a), is related to object selection without replacement. The number of elements in its sequence of invariants is equal to $\varnothing+1$.

TD2: This diagonal, associated with (7.1.b), is also related to object selection without replacement. The number of elements in its sequence of invariants is equal to the order of the parent sequence. The alleles in the range from TD2 to TD3 are systematic both in the sequence of counters and their number of elements reduce (or increase in the direction from TD3 to TD2) by "1". Hence, the interoperability in this range is based on the convolution product rule.

TD3: This diagonal is associated with a hybrid of (7.1.b) and (7.1.c). The size of the sequence of invariants in the process of reducements/conducement stays the same in both direction of TD3-TD4 or TD4-TD3. Though the reducements of the sequences of invariants are fully systematic, care is needed for the adjustment and the example clearly shows it. The alleles of TD3-TD4 are associated with the degree of the parent sequence stemming from its encapsulated direct products. Interoperability of the sequences of invariants in this range uses the direct product rule.

TD4: This diagonal is also unique and has no sequence of counters. The sequence of invariant has been transformed into the kernel, where the sum of its elements is minimum. A kernel also signifies that the sequence is conducemental.

Remark 8.3: The alleles in TD3-TD2 do not arise in all the conducemental sequences, unless the constituent product of the parent sequence involves at least one convolution/regeneration multiplication. 
Remark 8.4: Evidently, the operations for the tabular difference method are clumsy but its educational value in the application of conducemental algebra is recognized for making it possible to visualise the structure inherent in conducemental sequences.

Remark 8.5: Due to the constraint on the length of the paper, the author's findings on the negative spaces (the underworld) are not presented but the negative and positive worlds are intertwined.

\section{Conducement and Interoperability-Heuristic Rules and Applications}

Interoperability in conducemental algebra is presented by transforming the alleles in Example 7.1, as follows.

\subsection{Interoperability in the Conducement Direction}

The alleles in the conducemental direction (i.e. from TD4 to TD0) are operated using the following rules:

Rule 10.1: For Allele 0 (TD4), the kernel is primed by the following transformer: $\left\{\begin{array}{llllll}1 & 1 & 1 & 1 & 1 & 1 \\ 0 & 0 & 0 & 0 & 0\end{array}\right\}$, the most basic rule, and then " 0 " is replaced by " 1 " for each allele.

Rule 10.2: The transformer for TD1 is: $\left\{\begin{array}{llllll}1 & 1 & 1 & 1 & 1 & 1 \\ & 1 & 1 & 1 & 1 & 1\end{array}\right\}$ with the following flipping: 1 ) in the conducemental direction (TD4 to TD1), the first element becomes zero; 2) in the reducemental direction (TD1 to $\mathrm{TD} 4)$, the last element becomes zero. Interoperability of the alleles $(\mathrm{RR}=$ Regenerating Range, $\beta$ counts its alleles; CR = Convolution Range, $\alpha$ counts its alleles) is given as follows:

$$
\begin{aligned}
& \left.\left\langle 1 \begin{array}{lllllll}
\langle 1 & 7 & 4 & 0 & 0 & 0 & 0
\end{array}\right\rangle \oplus\left\{\begin{array}{lllllll}
1 & 1 & 1 & 1 & 1 \\
0 & 0 & 0 & 0 & 0
\end{array}\right\}\right\}={ }_{\beta=0}^{\mathrm{RR}}\left\{\begin{array}{lllllll}
1 & 7 & 4 & 0 & 0 & 0 & 0
\end{array}\right\} \\
& \left.\left\{\begin{array}{lllllll}
1 & 7 & 4 & 0 & 0 & 0 & 0
\end{array}\right\} \oplus\left\{\begin{array}{lllll}
1 & 1 & 1 & 1 & 1 \\
1 & 0 & 0 & 0 & 0
\end{array}\right\}\right\}={ }_{\beta=1}^{\mathbf{R R}}\left\{\begin{array}{lllllll}
1 & 8 & 4 & 0 & 0 & 0 & 0
\end{array}\right\} \\
& \left.\left\{\begin{array}{lllllll}
1 & 8 & 4 & 0 & 0 & 0 & 0
\end{array}\right\} \oplus\left\{\begin{array}{lllll}
1 & 1 & 1 & 1 & 1 \\
1 & 1 & 0 & 0 & 0
\end{array}\right]\right\}={ }_{\beta=2}^{\mathbf{R R}}\left\{\begin{array}{lllllll}
1 & 9 & 12 & 0 & 0 & 0 & 0
\end{array}\right\} \\
& \left.\left\{\begin{array}{lllllll}
1 & 9 & 12 & 0 & 0 & 0 & 0
\end{array}\right\} \oplus\left\{\begin{array}{lllll}
1 & 1 & 1 & 1 & 1 \\
1 & 1 & 1 & 0 & 0
\end{array}\right\}\right\}={ }_{\alpha=1}^{\mathrm{CR}}\left\{\begin{array}{lllllll}
1 & 10 & 21 & 12 & 0 & 0 & 0
\end{array}\right\} \\
& \left\{\begin{array}{lllllll}
1 & 10 & 21 & 12 & 0 & 0 & 0
\end{array}\right\} \oplus\left\{\begin{array}{llllll}
1 & 1 & 1 & 1 & 1 \\
1 & 1 & 1 & 1 & 0
\end{array} \quad 0\right\}={ }_{\alpha=2}^{\mathrm{CR}}\left\{\begin{array}{lllllll}
1 & 11 & 31 & 33 & 12 & 0 & 0
\end{array}\right\} \\
& \left.\left.\left\{\begin{array}{lllllll}
1 & 11 & 31 & 33 & 12 & 0 & 0
\end{array}\right\} \text { ( } \begin{array}{llllll}
1 & 1 & 1 & 1 & 1 \\
1 & 1 & 1 & 1 & 1
\end{array}\right\}\right\}=\left\{\begin{array}{lllllll}
1 & 12 & 31 & 42 & 64 & 12 & 0
\end{array}\right\} \\
& \left\{\begin{array}{lllllll}
1 & 12 & 42 & 64 & 45 & 12 & 0
\end{array}\right\} \oplus\left\{\begin{array}{llllll}
1 & 1 & 1 & 1 & 1 & 1 \\
1 & 1 & 1 & 1 & 1
\end{array}\right\} \\
& ={ }_{\alpha=4}^{\mathrm{CR}}\left\{\begin{array}{lllllll}
1 & 13 & 54 & 106 & 109 & 57 & 12
\end{array}\right\} \\
& \left\{\begin{array}{lllllll}
1 & 13 & 54 & 106 & 109 & 57 & 12
\end{array}\right\} \oplus\left\{\begin{array}{llllll}
0 & 1 & 1 & 1 & 1 & 1 \\
1 & 1 & 1 & 1 & 1
\end{array}\right\} \\
& ={ }^{\mathrm{TD} 0}\left\{\begin{array}{llllllll}
0 & 14 & 67 & 160 & 215 & 166 & 69 & 12
\end{array}\right\} \\
& \left\{\begin{array}{llllllll}
0 & 14 & 67 & 160 & 215 & 166 & 69 & 12
\end{array} \oplus \oplus\left\{\begin{array}{llllllll}
0 & 0 & 1 & 1 & 1 & 1 & 1 & 1 \\
& & 1 & 1 & 1 & 1 & 1
\end{array}\right\}\right. \\
& ={ }^{\mathrm{TD} 0}\left\{\begin{array}{lllllllll}
0 & 0 & 81 & 227 & 375 & 381 & 235 & 81 & 12
\end{array}\right\}
\end{aligned}
$$


It is noted that the size of the kernel in (9.1.a) is " 3 " and this size remains so until (9.1.c) but increases by " 1 " for each allele until (9.1.g). From here onwards, the size of non-zero elements in the diagonals remains constant. From (9.1.g) onwards, there is no more allele but a zero element is added for each trailing sequences. The presentation of the full rules for these dimensional parameters is beyond the remit of this paper.

\subsection{Interoperability in the Reducement Direction}

At the reducement direction, the operations are systematic and follow simple rules:

Rule 10.3: The transformer for the reducement is populated by the inverse of $\left\{\begin{array}{ll}1 & 1\end{array}\right\}$ but the number of the elements depends on its location with systematically changing their signs.

Rule 10.4: In the TD0 Range, the operations are carried out from the tailend of the transformer, but there is no problem with the normal operations.

Rule 10.5: At Transitional Diagonal 1, flipping occurs.

Rule 10.6: the number of the terms in the sequence of invariants is shrunk by 1 for each reducement in the TD2-TD3 range.

Rule 10.7: Each reducement in the TD3-TD4 Range replaces the last element by 1 .

The above are illustrated by the example below.

TD0: $\left.\begin{array}{lllllll}81 & 227 & 375 & 381 & 235 & 81 & 12\end{array}\right\} \oplus\left\{\begin{array}{cccccccc}1 & & & & & & \\ -1 & -1 & 1 & & & & \\ 1 & 1 & -1 & -1 & & & \\ -1 & -1 & 1 & 1 & 1 & -1 & -1 & 1 \\ 1 & 1 & -1 & -1 & 1 & 1 & \\ -1 & -1 & 1 & 1 & & & \\ 1 & 1 & & & & & \end{array}\right\}$

$=\left\{\begin{array}{lllllll}14 & 67 & 160 & 215 & 166 & 69 & 12\end{array}\right\}$

TD1: $\left\{\begin{array}{lllllll}14 & 67 & 160 & 215 & 166 & 69 & 12\end{array}\right\} \oplus\left\{\begin{array}{cccccccc}1 & & & & & & \\ -1 & -1 & 1 & & & & \\ 1 & 1 & -1 & -1 & 1 & & \\ -1 & -1 & 1 & 1 & -1 & -1 & 1 \\ 1 & 1 & -1 & -1 & 1 & 1 & \\ -1 & -1 & 1 & 1 & & \\ 1 & 1 & & & & & \end{array}\right\}$

TD2 (TD2-TD3.1):

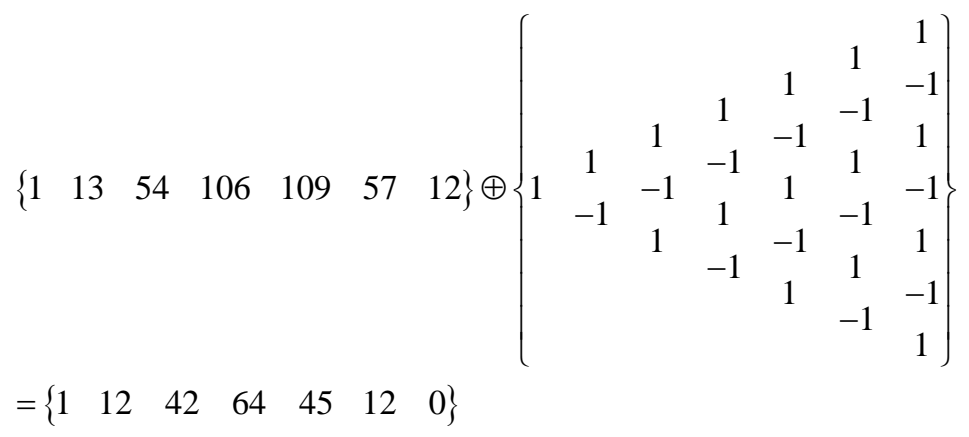

TD2-TD3.2: 


$$
\begin{aligned}
& \left\{\begin{array}{lllllll}
1 & 12 & 42 & 64 & 45 & 12 & 0
\end{array}\right\} \oplus\left\{\begin{array}{ccccccc} 
& & & & 1 & \\
& & & 1 & 1 & -1 \\
& 1 & 1 & -1 & -1 & 1 & \\
1 & -1 & 1 & 1 & -1 & 0 \\
-1 & 1 & -1 & -1 & 1 & \\
& & & & 1 & -1
\end{array}\right\} \\
& =\left\{\begin{array}{lllllll}
1 & 11 & 31 & 33 & 12 & 0 & 0
\end{array}\right\}
\end{aligned}
$$

TD2-TD3.3:

$$
\begin{aligned}
& \left\{\begin{array}{lllllll}
1 & 11 & 31 & 33 & 12 & 0 & 0
\end{array}\right\} \oplus\left\{\begin{array}{cccccc}
\multicolumn{9}{c}{1} & 1 & & \\
& 1 & 1 & -1 & -1 & \\
1 & -1 & 1 & 1 & 0 & 0 \\
-1 & 1 & & -1 & \\
& & & -1 & 1 &
\end{array}\right\}=\left\{\begin{array}{lllllll}
1 & 10 & 21 & 12 & 0 & 0 & 0
\end{array}\right\} \\
& \text { TD2-TD3.4: }\left\{\begin{array}{lllllll}
1 & 10 & 21 & 12 & 0 & 0 & 0
\end{array}\right\} \oplus\left\{\begin{array}{ccccccc}
1 & 1 & & & & \\
1 & -1 & -1 & 0 & 0 & 0 \\
-1 & 1 & 1 & & &
\end{array}\right\}=\left\{\begin{array}{lllllll}
1 & 9 & 12 & 0 & 0 & 0 & 0
\end{array}\right\} \\
& \text { TD3-TD4.1: }\left\{\begin{array}{lllllll}
1 & 9 & 12 & 0 & 0 & 0 & 0
\end{array}\right\}\left\{\begin{array}{cccccc}
1 & 1 & & & \\
1 & -1 & 0 & 0 & 0 & 0 \\
-1 & 1 & & & &
\end{array}\right\}=\left\{\begin{array}{lllllll}
1 & 8 & 4 & 0 & 0 & 0 & 0
\end{array}\right\}
\end{aligned}
$$

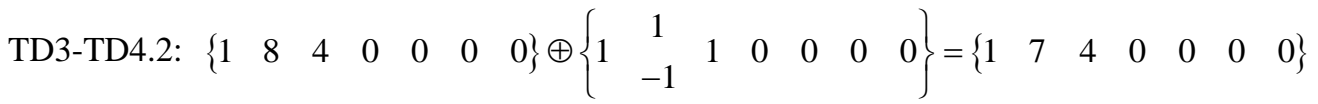

$$
\begin{aligned}
& \text { TD4: }\left\{\begin{array}{llllllll}
1 & 7 & 4 & 0 & 0 & 0 & 0
\end{array}\right\} \oplus\left\{\begin{array}{lllllll}
1 & 1 & 1 & 0 & 0 & 0 & 0
\end{array}\right\}=\left\langle\begin{array}{lllllll}
1 & 7 & 4 & 0 & 0 & 0 & 0
\end{array}\right\}
\end{aligned}
$$

Remark 9.1: The logic of (9.2.a) to (9.2.g) is obvious and follows the convolution range but it changes to a different logic appropriate to the regeneration product rule to (9.2.h) and (9.2.i).

Remark 9.2: The above unearths encapsulation in an elegant and astounding way.

\section{Beyond the Examples Presented}

Avigad [3] discussed the role of different approaches in treating the same problem in mathematics, according to whom this is the problem of multiple proofs. He argues that "On the standard account, the value of a mathematical proof is that it warrants the truth of the resulting theorem. Why, then, is it often the case that a new proof of a theorem is often highly valued?" His response is that each proof provides new insights and the proof of a theorem does something more than establishing the truth of the results. Arithmetic calculus may be considered as one such case.

The tip of the iceberg is the nearest expression that can characterise the new operators and notations in the arithmetic calculus. The above is a basic introduction without any significant demonstration of its scope and problem-solving strength, as these will be presented in a series of papers by the author in the near future. However, one example is shown below to show that arithmetic calculus is not trivial, as follows:

Example 10.1: Recast first kind Stirling Numbers by arithmetic calculus to the following operator:

$$
\left\{\begin{array}{lllll}
t_{1} & t_{2} & t_{3} & \cdots & t_{k}
\end{array}\right\} \hat{\oplus}\left\{\begin{array}{llllll}
1 & 2 & 3 & \ldots & k & 1 \\
1 & 1 & & 1
\end{array}\right\}
$$


Consider the case $K=4$, for which the sequence is: $\left\{\begin{array}{llll}1 & 7 & 6 & 1\end{array}\right\}$, thus:

$$
\begin{aligned}
& \left\{\begin{array}{llll}
1 & 7 & 6 & 1
\end{array}\right\} \hat{\oplus}\left\{\begin{array}{lllll}
1 & 2 & 3 & 4 & \\
1 & 1 & 1 & 1
\end{array}\right\} \\
& =\left\{\begin{array}{lllll}
1 \times 1 & 1 \times 1+7 \times 2 & 7 \times 1+6 \times 3 & 6 \times 1+1 \times 4 & 1 \times 1
\end{array}\right\} \\
& =\left\{\begin{array}{lllll}
1 & 15 & 25 & 10 & 1
\end{array}\right\}
\end{aligned}
$$

Arithmetic calculus is highly flexible and applicable to any conducemental sequences. The author has applied it to a wide range of circumstances and has established their overall patterns but they are beyond the remit of this single paper. One aspect that can be reflected in passing is the versatility of this calculus in treating some irregularities or mutation in conducemental sequences. This is illustrated by the basic sequence of natural numbers and the perturbation of its $1^{\text {st }}$ and $2^{\text {nd }}$ terms:

$$
\begin{gathered}
\left\{\mathcal{R} \mid\left\{\begin{array}{llllll}
1 & 2 & 3 & 4 & \cdots & n
\end{array}\right\}\right\}=\{1\} \odot\left\{\left(\begin{array}{c}
n \\
n-1
\end{array}\right)\right\} \\
\left\{\mathcal{R} \mid\left\{\begin{array}{llllllll}
2 & 2 & 2 & 3 & 4 & 5 & \cdots & n
\end{array}\right\}\right\}=\left\{\begin{array}{llll}
2 & -2 & 0 & 1
\end{array}\right\} \odot\left\{\left(\begin{array}{c}
n \\
n-1
\end{array}\right)\left(\begin{array}{c}
n-1 \\
n-2
\end{array}\right) 0\left(\begin{array}{c}
n-2 \\
n-4
\end{array}\right)\right\}
\end{gathered}
$$

Remark 10.1: Note that and ${ }^{+}\left\langle\begin{array}{llll}2 & -2 & 0 & 1\end{array}\right\rangle=1$ and therefore perturbation does not alter the true kernel value.

The reality behind arithmetic calculus is yet to be presented by the author. If algebraic calculus refers to independent variables such as space and time, the space in arithmetic calculus refers to building blocks in particulate media and time is transformed into generations. Rules are no longer in terms of differentials but explicit values selected by experience. This is a philosophy that will be presented by the author soon.

The possibilities with arithmetic calculus are countless and each conducemental sequence can be identified by its kernel at a specific level, as well as by its sequence of counters. The kernels are the signatures (as DNAs) of conducemental sequences and may be used to devise the "Tree of Numbers" for a whole family of the sequences of natural numbers, each with a completely known property. This would integrate existing knowledge on number sequences, which can dwarf the tree of life. An indicative Tree of Numbers suggested by the author [2] is illustrated in Figure 1. This is the key to interconnectivity in sequences of natural numbers, without which their knowledge seems disconnected. Tree of Numbers can be a powerful and versatile tool, going deep to the roots of mathematics.

Number sequences have received attention for many millennia with the intensity of developments increasing since the introduction of generating functions by De Moire in 1730. Therefore, this paper is at risk of an instant reaction of lacking novelty, as the subject is old and existing solutions are mature, to the extent that further developments may be regarded as "the Emperor's New Clothes". However, this paper hopefully shows that arithmetic calculus leads to increased knowledge and simpler operations and therefore such a concern is trivial. If arithmetic calculus is dismissed, the least harmful outcomes would be the sustenance of ongoing clumsy algebraic operations and the overlooking of simple and systematic operations to say the least.

At a serious note, the case of deconvolution presents a serious case for the application of arithmetic calculus. Also the application to conducemental sequences shows that indeed the single most important difference between conducemental algebra and conventional algebra is the role of rules. Through conducemental algebra, rules assume an explicit role and a due place in mathematics in the form of sequence of counters. Conventional algebra is oblivious to existence of such rules, or at least, it is not minded to provide identity for such an important concept. Instead ad hoc procedures have been invented, which reflect the ingenuity of the mathematicians but not the versatility of mathematics. The presentation in this paper is necessarily heuristic but other papers are ready to present theorems or axioms once this barrier is passed.

Another direction of the author's research is the generalisation of theory of evolution (GTE), in which the ability to break down any evolving entity into building blocks and rule is pivotal. This invoked the author to seek the dimensionalities of evolutionary processes, based on neo-Darwinism. Surprisingly, all the attributes in the GTE have their traces in arithmetic calculus. The author is unable to present his ideas on the GTE without first publishing the arithmetic calculus and hence this paper is pivotal towards a larger aim. 


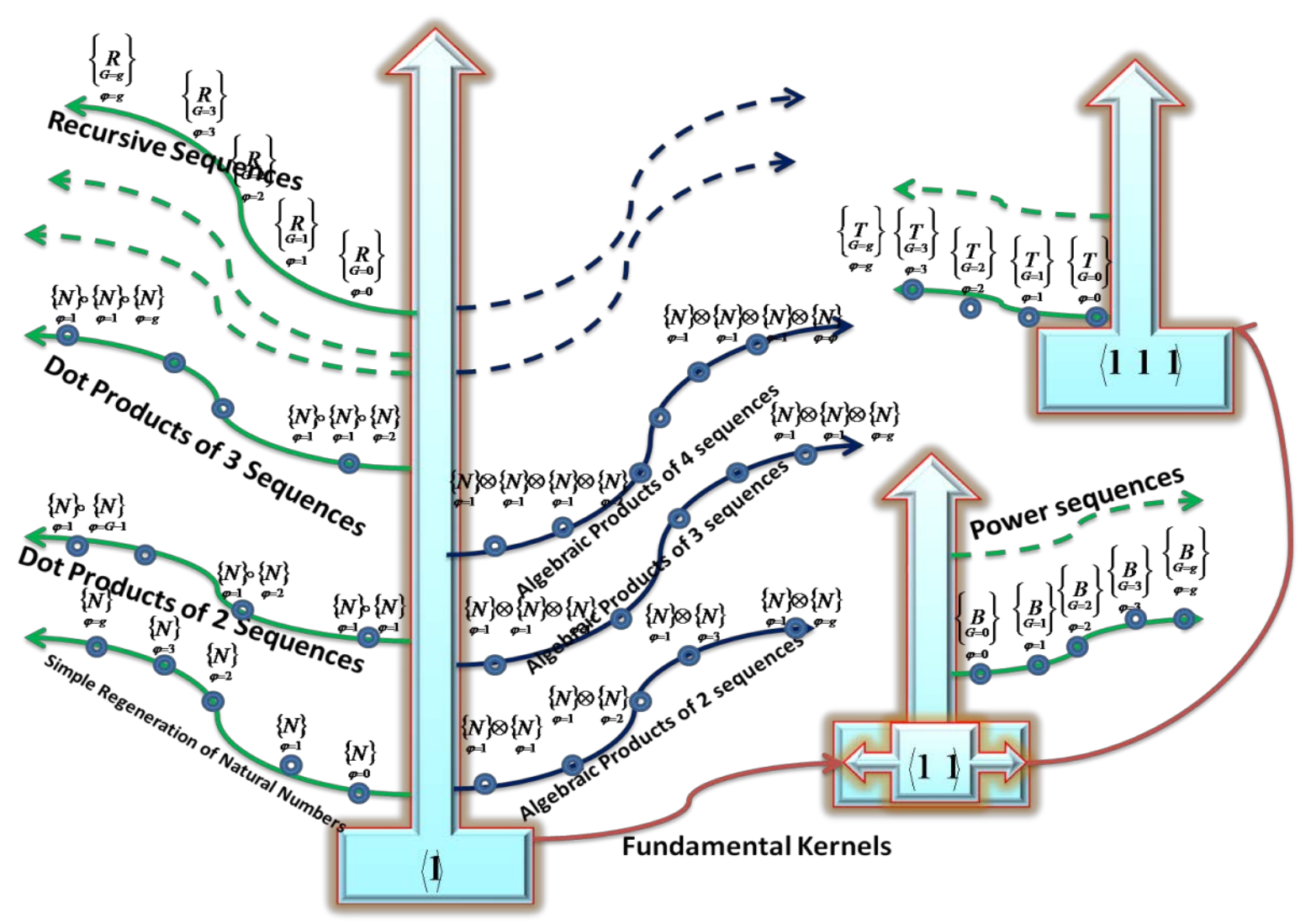

Figure 1. A prototype of tree of numbers.

\section{Conclusions}

This paper promotes new ways of thinking on 1) shifting the focus from sets/sequences of numbers to their elements; and 2) show that each element has a hidden structure that can be unearthed. This structure is handled by arithmetic calculus and conducemental algebra. Conventional algebra applied to sets/sequences of numbers based on natural number retards mathematics: the problems become unduly complicated; operations cannot be systematised similar to matrix-type operations; the potential for different methods of multiplication of two different sequences have not been transformed into classic operators. Arithmetic calculus and conducemental algebra overcome these problems and are surprisingly extremely simple and blatantly self-obvious. They are powerful and can possibly provide a framework to overhaul conventional algebra of sequences of natural numbers.

There is one disadvantage with using number sequences, because the subject is familiar and therefore their treatments may seem as the reinvention of the wheel. However, this is not true as the paper presents a new calculus but the classic sequences of natural numbers are used to show the effectiveness of the new calculus. This calculus attacks the problems at their roots by factorisation by the virtue of new operators: 1 ) direct products, by which the elements of a sequence are raised to a power in a consistently definable manner-this is one of the mechanisms to create complexity; 2) regenerating products with the effect of adding layer upon layers to open sequences without adding to their complexity; 3) convoluting products, as in regenerating products but the operations are carried out for closed sequences; 4) algebraic products.

Arithmetic calculus is applied to a family of sequences of natural numbers, to be referred to as the conducemental sequences. As such, the study of these sequences is old but enough is presented in this paper to justify that there is a missing calculus, as these sequences are treated differently by arithmetic calculus. Conducemental sequences are shown at a heuristic level to have a total interoperability and even school students can cope with most, if not all, of these mathematical operations. The whole operations can be automated. The author's emphasis is that the need for the new calculus is not for the sake of it but to fill a gap. 


\section{Acknowledgements}

The development in this paper is a contribution to open scientific and mathematical culture. No moral right is given to any application of the emerging calculus for any development, software or otherwise, that uses the new capability and closes their outcome to the public. The author strongly supports open science/mathematics in every respect.

\section{References}

[1] Wilf, H.S. (1994) Generating Functionology. http://www.math.upenn.edu/ wilf/gfology2.pdf

[2] Khatibi, R. (2013) Chapter 1: Learning from Natural Selection in Biology: Reinventing Existing Science to Generalise Theory of Evolution-Evolutionary Systemics. In: Lynch, J.R., Derek, T. and Williamson, D.T., Eds., Natural Selection: Biological Processes, Theory and Role in Evolution, 1-96.

https://www.novapublishers.com/catalog/product_info.php?products_id=41521

[3] Avigad, J. (2010) Understanding, Formal Verification, and the Philosophy of Mathematics. http://www.andrew.cmu.edu/user/avigad/Papers/understanding2.pdf 


\section{Glossary}

Algebraic products

Alleles

Alleles in

Convolution Range

Alleles in

Regeneration Range

Arithmetic calculus

Base

Building blocks

Complexity

Complexity gain

Conducement

Conducemental
This is defined by (4.10) in Section 4.6.

It is denoted by: $\{\cdot\} \otimes\{\cdot\}$. It is a composite but not explored in any greater detail in this paper.

Similar to chemistry, different configurations of the same sequence.

In complex synthetic sequences of natural numbers, alleles are in two ranges: in the Convolution Range(CR) and Regeneration Ranges (RR). Alleles in CR are outcomes of convolution products and are encapsulated and directly related to the encapsulated generation of the parent sequence. They are denoted as:

$$
\left\{\alpha_{0}^{\mathrm{CR}} \mid \cdot\right\}
$$

where $\alpha$ denotes allele, its superfix CR signifies convolution range.

In complex synthetic sequences of natural numbers, alleles are in two ranges: in the Convolution Range(CR) and Regeneration Ranges (RR). Alleles in RR are outcomes of direct products and are encapsulated and directly related to the encapsulated degree of the parent sequence. They are denoted

$$
\left\{\alpha_{i}^{\mathrm{RR}} \mid \cdot\right\}
$$

where $\alpha$ denotes allele, its superfix RR signifies the range and the suffix $i$ signifies its allele position. Note that, these alleles are in the regenerating zone and their interoperability operations are rather complicated.

A term proposed to suggest a systematic approach to summing a family of sequences based on natural numbers.

In combinatoric operators, the base refers to the smaller finite and distinct objects selection from larger ones (which is referred to as the level).

Another term for kernel.

The core of a sequence when it is reduced to its rock bottom, related to kernel.

The kernel of the sequence of natural numbers: $\left\{\begin{array}{lll}1 & 2 & \cdots\end{array}\right\}$ is $\langle 1\rangle$ and therefore its complexity by definition is 1 . Any other synthetic sequence of natural numbers with a greater complexity has a gain $=$ its complexity size -1

The operation of systematically summing the terms of a sequence together.

The term is proposed in lieu of summing or integration.

Different notations are used for conducement:

$$
\{\operatorname{Con} \mid\{\cdot\}\} \text { or }{ }^{c}\{\cdot\} \text { or }{ }^{+}\{\cdot\}
$$

This is a mathematical expression, which expresses the sum. For instance:

$$
\left.\left\{\begin{array}{ccccc}
c & 2 & 3 & \cdots & \cdots \\
n-1
\end{array}\right)\right\}=\left(\begin{array}{c}
n+1 \\
n-1
\end{array}\right) .
$$

Its conducemental is: $\left(\begin{array}{l}n+1 \\ n-1\end{array}\right)$. A conducemental is easily obtained.

Conducemental direction In the Difference table, this is the direction along which.

Convolution products

This is defined by (4.6) in Section 4.4.

It is denoted by: $\{\cdot\} \hat{\oplus}\{\cdot\}$. The accent on $\oplus$ signifies that the products are finite and dipping.

Degree

The degree of a sequence is directly related to the effect of using the direct product rule. Some of the rules are presented in heuristic rules terms. It is an important dimension of arithmetic calculus.

This age old method is given a new lease of life when the sequence of counters is superimposed on its diagonals. This paper show in Section 7 that the Difference Table has many generic zones and offers appropriate terms to describe them. 


\section{Continued}

Direct product

Encapsulation

Generation

Interoperability

Leading terms

Leading zone

Kernel

Level

Natural numbers

Order

Priming

Parent sequence

Recursive sequences

Reducement

Reducemental

Reducemental analysis

Regeneration

Regenerating Zone

Regenerating product
This is defined by (4.2) and (4.3) in Section 4.2. It is denoted by two different approaches: $\{\cdot\} \circ\{\cdot\}$ when the product are a sequence $\{\cdot\} \odot\{\cdot\}$ when the products are conducemental. This may equally be called the dot product rule, as the symbols suggests.

When a set of multiplication operation has been carried out, the emerging parent sequence encapsulates its inherent complexity. This can be analysed by reducemental analysis in terms of its order, which is composed of encapsulated degree, encapsulated generation. When a parent sequence is re-operated by multiplication, it creates layers of encapsulation. In general, reducemental analysis is capable of keeping the track of the last generation and degree.

Generation is an important dimension of arithmetic calculus and refers to the number of times that a sequence is regenerated.

Arithmetic calculus unearths a highly interoperable world of mathematics. The role of the sequences of counter is normally self-evident when the interconnectivity of one sequence is sought from another one. However, this is not so for the sequences of invariants. This leads to another set of very interesting operations, termed in the paper as interoperability.

Consider the finite sequence: $\left\{\begin{array}{lllll}1 & 15 & 25 & 10 & 1\end{array}\right\}$. Its first two terms are increasing towards a maximum and then dipping to a lower value again. The increasing terms are referred to as the leading terms and the dipping terms as the trailing terms.

One of the zones among the many zones in Difference Table as delineated in Table 7.1 in generic terms. This is located on the reducemental direction of Transitional Diagonal 1.

A kernel is not a sequence but the very final residue of a sequence when subjected to generations of reducement. Kernels measure the complexity of a sequence.

Another term to kernel is building blocks.

In combinatoric operator, the level refers to the finite and distinct objects, from which a smaller set is selected (this is referred to as the base).

This is simply the numbers as used in natural language. They are $1,2,3, \cdots$

The order of a combinatoric operator is the difference between its level and its base. It combined degree and generation, the three of which are the key for specifying a sequence.

Conversion of a kernel to a sequence, where the kernel is not a sequence yet.

This is the top line in Difference Table.

Recursive sequences are incomplete sequences and often look like the work in hand but more is expected to be done.

This term is introduced in this paper and is equivalent to differentiation.

This term is introduced in this paper and is equivalent to genetics of living things.

This is equivalent to dimensional analysis in physical sciences. Through this analysis, an overarching view is taken of a sequence of natural numbers but the relevant analysis has not been detailed in this paper.

This is a variation of convolution, in which there is no dipping. The regeneration operator is defined by (4.6) in Section 4.1.2.

This refers to alleles located between Transitional Diagonal 2 and 3.

In this zone, the size of the allele remain constant.

This is defined by (4.4) in Section 4.3.

It is denoted by: $\{\cdot\} \oplus\{\cdot\}$. 


\section{Continued}

Sequence of counters

Sequence of invariants

Sequence

Sequences of natural numbers

Series

Size

Space

Specification

Synthetic sequences of natural numbers

Zone
The paper heuristically states that each synthetic sequence of natural numbers can be factorised. Into two sequences. One of these sequences is the sequence of counters. This sequence embodies the rules and it can be a selection of even any arbitrary operations. The simplest sequence of counters is that in which all the elements are unity.

The paper heuristically states that each synthetic sequence of natural numbers can be factorised. Into two sequences. One of these sequences is the sequence of invariants. This sequence embodies the building blocks but organised in the manner of a sequence. The simplest sequence of invariants is $\{1\}$, which can be primed from: kernel: $\langle 1\rangle$.

A sequence of numbers is a collection of highly ordered set of individual numbers, each referred to as an element or a term, separated by a coma or space. A sequence is denoted by a curly bracket: $\left\{\begin{array}{lll}\cdot & \cdot\end{array}\right\}$.

The terms or elements of a sequence are either separated from one another by space or by a coma. The terms sequence and series are not interchangeable in this paper.

Natural numbers are a product of natural selection at the cultural level and without any real concern to mathematics. However, the sequence of natural numbers is a declaration to mathematise natural numbers. This paper shows that the sequence of natural numbers are themselves a synthesis, primed out of the kernel $\langle 1\rangle$.

It is a sequence with prescribed operation on all of its terms.

The Size of a sequence/series is the number of elements in it and this is normally reflected in the ultimate term, often denoted by $n$. For an infinite sequence/series, the size is infinite but still its last term may be denoted as $n$ to reflect the point of interest.

Difference Table is broadly deciphered in terms of

1) Space: which refers to the horizontal layout of the elements of the parent sequences or their derivatives.

2) Zones: which refers to the diagonal layout of the sequences of counters.

Note that these terms are not deliberately in this paper.

The paper unravels that each synthetic sequence of natural numbers.

Although the operators for regeneration and raising to powers have not been presented yet, these two operations have a direct bearing on the order of a sequence. These three terms are not interchangeable, as each represents a specific concept. Arithmetic calculus proposes a notation for generation, degree and order of a sequence.

The paper holds that there are countless sequences synthesised from the sequence of natural numbers.

Four TDs are distinguished and they are numbers in the reducemental direction:

TD1: delineates the trailing zone from the leading zone.

Transitional Diagonal (TD) TD2: delineate the standard allele.

TD3: delineates the transition from convoluting zone to regenerating zone.

TD4: delineates the transition from the regenerating zone to the kernel, where there is no more sequence but plain building blocks.

Difference Table has many zones and these are delineated in Table 7.1 in generic terms. Each zone has been given a name and defined in this Glossary. A range of generic diagonals are distinguished in terms of Transitional Diagonals 1, 2, 3 and 4. 
Scientific Research Publishing (SCIRP) is one of the largest Open Access journal publishers. It is currently publishing more than 200 open access, online, peer-reviewed journals covering a wide range of academic disciplines. SCIRP serves the worldwide academic communities and contributes to the progress and application of science with its publication.

Other selected journals from SCIRP are listed as below. Submit your manuscript to us via either submit@scirp.org or Online Submission Portal.
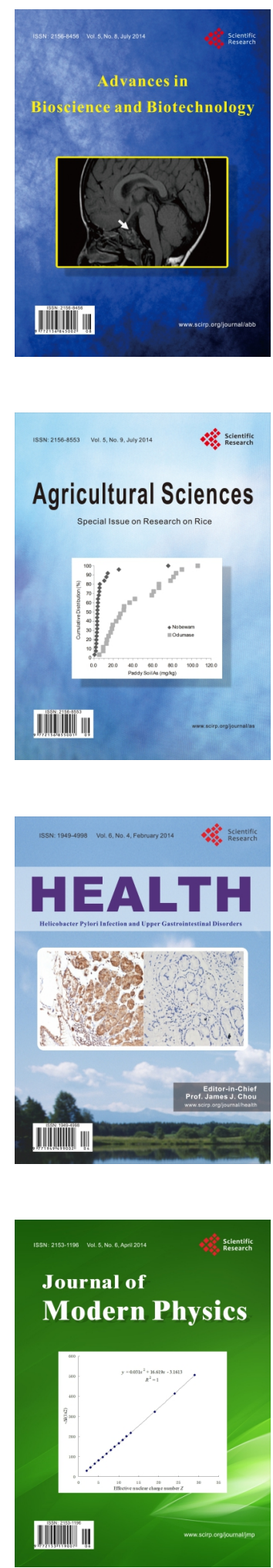
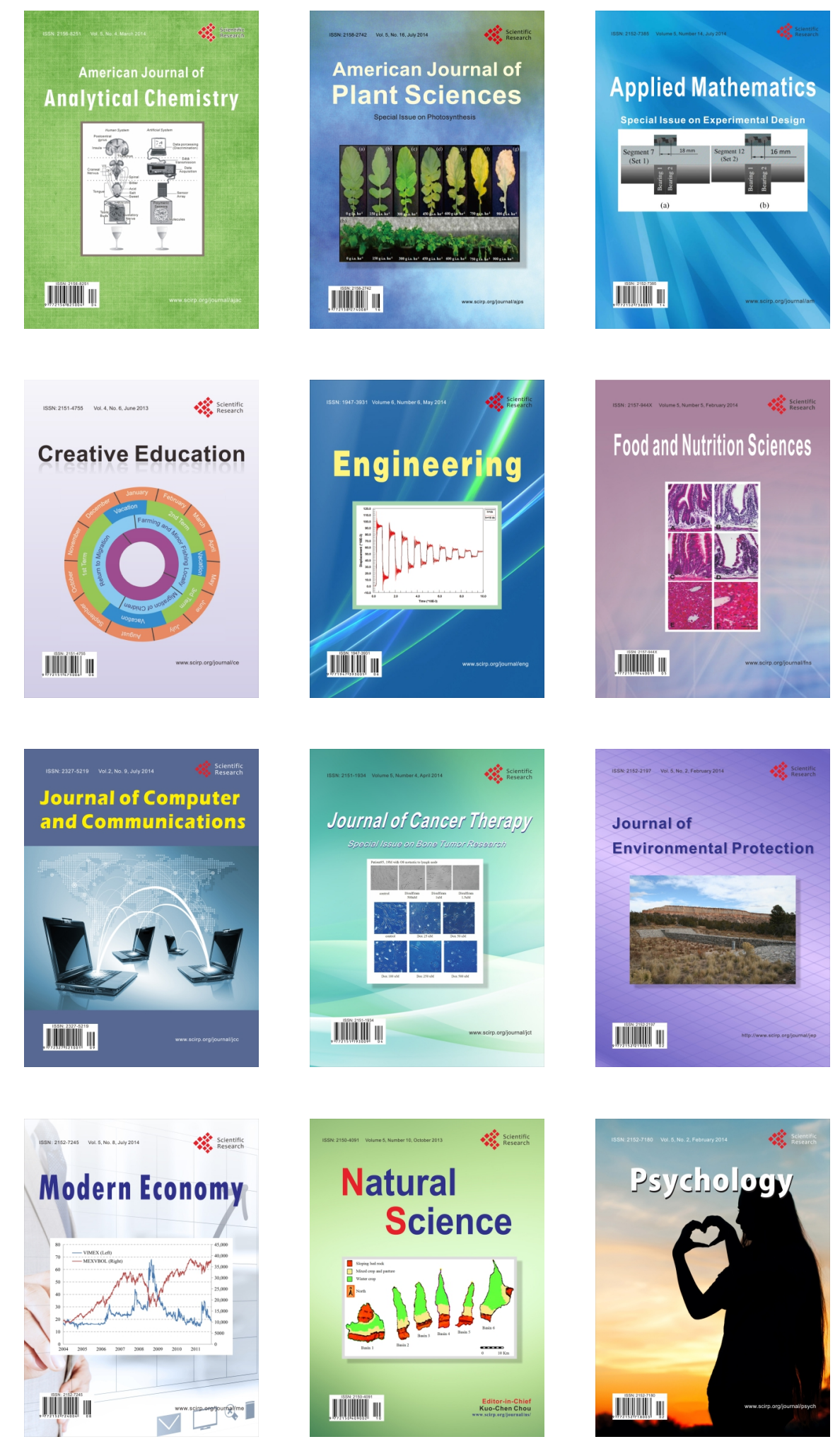